\title{
NUMERICAL ANALYSIS OF TURBULENT FLOW AROUND TWO- DIMENSIONAL BODIES USING NON-ORTHOGONAL BODY-FITTED MESH
}

\author{
Md. SHAHJADA TARAFDER* ${ }^{*}$ and M. Al MURSALINE \\ Department of Naval Architecture and Marine Engineering \\ Bangladesh University of Engineering and Technology \\ Dhaka - 1000, BANGLADESH \\ E-mail: shahjada68@yahoo.com
}

\begin{abstract}
This paper deals with the numerical simulation of a turbulent flow around two-dimensional bodies by the finite volume method with non-orthogonal body-fitted grid. The governing equations are expressed in Cartesian velocity components and solution is carried out using the SIMPLE algorithm for collocated arrangement of scalar and vector variables. Turbulence is modeled by the $k$ - $\varepsilon$ turbulence model and wall functions are used to bridge the solution variables at the near wall cells and the corresponding quantities on the wall. A simplified pressure correction equation is derived and proper under-relaxation factors are used so that computational cost is reduced without adversely affecting the convergence rate. The numerical procedure is validated by comparing the computed pressure distribution on the surface of NACA 0012 and NACA 4412 hydrofoils for different angles of attack with experimental data. The grid dependency of the solution is studied by varying the number of cells of the C-type structured mesh. The computed lift coefficients of NACA 4412 hydrofoil at different angles of attack are also compared with experimental results to further substantiate the validity of the proposed methodology.
\end{abstract}

Key words: turbulent flow, finite volume method, pressure distribution.

\section{Introduction}

Turbulence being so ubiquitous in nature has its prominent influence in almost all practical flows, thus making its computation so important for applied mathematicians and engineers.

However, the fluid mechanics phenomena in the turbulent flow regime are interesting and at the same time considerably more complicated. In addition to the inherent complicacies of the system of partial differential equations governing fluid flow, turbulence and geometric complexities of the domain give rise to further challenges. While turbulence may be dealt with using a suitable turbulence model, domain complexity may be circumvented using body fitted coordinates. In the past, various methods were employed to overcome these difficulties associated with numerical computation of turbulent flow in complex domains. Rhie [1] used finite volume method for the solution of two-dimensional incompressible, steady turbulent flows over airfoils using $k-\varepsilon$ turbulence model and wall functions. Instead of staggered grids, the body fitted grid utilized a collocated arrangement of variables where the false pressure field was avoided by special momentum interpolation. Peric [2] developed a finite volume method for viscous flow in complex geometries, discretizing the governing transport equations in terms of Cartesian vector and tensor components and arbitrary non-orthogonal coordinates.

Demirdzic et al. [3] provided a complete exposition of a finite volume approach to the calculation of turbulent flows. Karki and Patankar [4] presented a general calculation procedure for computing fluid flow and related phenomenon in arbitrary-shaped domains with physical covariant velocity components selected

\footnotetext{
* To whom correspondence should be addressed
} 
as the dependent variables in momentum equations and the coupling between the continuity and momentum equations ensured using the SIMPLE algorithm.

Majumdar [5] reported that solutions of steady-state problems from Rhie and Chow momentum interpolation are dependent on the underrealxation factor. Choi [6] reported that the solution using the original Rhie and Chow scheme is time step size dependent. He proposed a modified Rhie and Chow scheme for an unsteady problem which is quite similar to the scheme for a steady problem used by Majumdar.

Moreover, Masuko and Ogiwara [7] carried out numerical simulation of viscous flow around ships having practical hull forms. The governing equations were discretized by finite difference approximation and solved with SIMPLE algorithm adopting the $k-\varepsilon$ turbulence model and standard wall functions. Yu et al. [8] discussed different momentum interpolation practices for collocated grid systems. Mulvany et al. [9] carried out an assessment of two-equation turbulence models for high Reynolds number hydrofoil flows using the finite volume method and SIMPLE solution technique.

Kuzmin and Mierka [10] presented a detailed numerical study of the $k$ - $\varepsilon$ turbulence model using algebraic flux correction to enforce the positivity constraint. Emphasis was laid on a new implementation of wall functions whereby the boundary conditions for $\mathrm{k}$ and $\varepsilon$ were prescribed in a weak sense. Demirdzic [11] discussed the discretization of diffusion term in finite volume continuum mechanics. Martınez et al. [12] proposed a possible correction for under-relaxation factor dependency in the Original Momentum Interpolation Method (OMIM).

The aim of the present paper is to simulate the turbulent flow around two-dimensional bodies by the finite volume method with non-orthogonal body fitted grid. The $k$ - $\varepsilon$ turbulence model and wall functions are used to bridge the solution variables at the near wall cells and the corresponding quantities on the wall. The solution is carried out using the SIMPLE algorithm with a simplified pressure correction equation for collocated arrangement for scalar and vector variables. Despite using a simplified pressure correction equation to reduce computational cost, and facilitate the solution of linear systems, a satisfactory convergence rate is achieved by proper choice of under-relaxation factors. The pressure distribution and lift forces on the surface of the hydrofoils far away from the free surface are compared with the experiment and the agreement is found to be quite satisfactory which reflects the accuracy of the present numerical methodology.

\section{Mathematical modelling of fluid flow problem}

\subsection{Governing equations}

In the Cartesian co-ordinate system, the steady two-dimensional turbulent flow around a hydrofoil for an incompressible fluid is governed by the following time-averaged equations

$$
\begin{aligned}
& \frac{\partial u}{\partial x}+\frac{\partial v}{\partial y}=0 \\
& \frac{\partial(\rho u u)}{\partial x}+\frac{\partial(\rho u v)}{\partial y}=\frac{\partial}{\partial x}\left[\left(\mu+\mu_{T}\right) \frac{\partial u}{\partial x}\right]+\frac{\partial}{\partial y}\left[\left(\mu+\mu_{T}\right) \frac{\partial u}{\partial y}\right]-\frac{\partial P}{\partial x}, \\
& \frac{\partial(\rho u v)}{\partial x}+\frac{\partial(\rho v v)}{\partial y}=\frac{\partial}{\partial x}\left[\left(\mu+\mu_{T}\right) \frac{\partial v}{\partial x}\right]+\frac{\partial}{\partial y}\left[\left(\mu+\mu_{T}\right) \frac{\partial v}{\partial y}\right]-\frac{\partial P}{\partial y}
\end{aligned}
$$

where $u$ and $v$ are the mean velocity components in the $x$ and $y$ directions, respectively, $\rho$ is the fluid density, $P$ is the mean pressure and $\mu$ is the laminar viscosity. $\mu_{T}$ is the turbulent viscosity and is given by 


$$
\mu_{T}=C_{\mu} \rho \frac{k^{2}}{\varepsilon}
$$

where $k$ is the turbulent kinetic energy, $\varepsilon$ is the dissipation rate of $k$ and $C_{\mu}$ is a constant. In the $k-\varepsilon$ turbulence model, $k$ and $\varepsilon$ are governed by the following transport equations in the Cartesian coordinate system

$$
\begin{aligned}
& \frac{\partial(\rho u k)}{\partial x}+\frac{\partial(\rho v k)}{\partial y}=\frac{\partial}{\partial x}\left[\left(\mu+\frac{\mu_{T}}{\sigma_{k}}\right) \frac{\partial k}{\partial x}\right]+\frac{\partial}{\partial y}\left[\left(\mu+\frac{\mu_{T}}{\sigma_{k}}\right) \frac{\partial k}{\partial y}\right]+G-\rho \varepsilon, \\
& \frac{\partial(\rho u \varepsilon)}{\partial x}+\frac{\partial(\rho v \varepsilon)}{\partial y}=\frac{\partial}{\partial x}\left[\left(\mu+\frac{\mu_{T}}{\sigma_{\varepsilon}}\right) \frac{\partial \varepsilon}{\partial x}\right]+\frac{\partial}{\partial y}\left[\left(\mu+\frac{\mu_{T}}{\sigma_{\varepsilon}}\right) \frac{\partial \varepsilon}{\partial y}\right]+\frac{\varepsilon}{k}\left(C_{\varepsilon 1} G-C_{\varepsilon 2} \rho \varepsilon\right)
\end{aligned}
$$

where $G$ is the production of $k$ and is given by

$$
G=2 \mu_{T}\left[\left(\frac{\partial u}{\partial x}\right)^{2}+\left(\frac{\partial v}{\partial y}\right)^{2}\right]+\mu_{T}\left(\frac{\partial u}{\partial y}+\frac{\partial v}{\partial x}\right)^{2}
$$

The standard values of the constants in Eqs (2.4), (2.5) and (2.6) are as follows

$$
C_{\mu}=0.09, \quad \sigma_{k}=1, \quad \sigma_{\varepsilon}=1.3, \quad C_{\varepsilon 1}=1.44, \quad C_{\varepsilon 2}=1.92 .
$$

Equations (2.1), (2.2), (2.3), (2.5) and (2.6) may be represented in the following generic form

$$
\frac{\partial(\rho u \Phi)}{\partial x}+\frac{\partial(\rho v \Phi)}{\partial y}=\frac{\partial}{\partial x}\left[\Gamma \frac{\partial \Phi}{\partial x}\right]+\frac{\partial}{\partial y}\left[\Gamma \frac{\partial \Phi}{\partial y}\right]+R^{\Phi}(x, y)
$$

where $u$ and $v$ are the mean velocity components, $\Phi$ is any generic dependent variable $(u, v, k, \varepsilon), \Gamma$ is an effective diffusion coefficient and $R^{\Phi}$ is the source term. Note that for continuity equation $\Phi=1, \Gamma=0$, $R^{\Phi}=0$, for $u$-momentum equation $\Phi=u, \Gamma=\mu+\mu_{T}, R^{\Phi}=-\frac{\partial P}{\partial x}$ and so on. Considering the body fitted co-ordinate system, $\xi=\xi(x, y), \eta=\eta(x, y)$ as shown in Figs 1 (a and b) Eq.(2.9) can be transformed into the following form

$$
\begin{aligned}
& \frac{1}{J} \frac{\partial(\rho U \Phi)}{\partial \xi}+\frac{1}{J} \frac{\partial(\rho V \Phi)}{\partial \eta}= \\
& =\frac{1}{J} \frac{\partial}{\partial \xi}\left[\frac{\Gamma}{J}\left(\alpha \frac{\partial \Phi}{\partial \xi}-\beta \frac{\partial \Phi}{\partial \eta}\right)\right]+\frac{1}{J} \frac{\partial}{\partial \eta}\left[\frac{\Gamma}{J}\left(\gamma \frac{\partial \Phi}{\partial \eta}-\beta \frac{\partial \Phi}{\partial \xi}\right)\right]+S^{\Phi}(\xi, \eta)
\end{aligned}
$$

where

$$
U=u \frac{\partial y}{\partial \eta}-v \frac{\partial x}{\partial \eta}, \quad V=v \frac{\partial x}{\partial \xi}-u \frac{\partial y}{\partial \xi}
$$

are the contra variant velocity components. 


$$
\alpha=\left(\frac{\partial x}{\partial \eta}\right)^{2}+\left(\frac{\partial y}{\partial \eta}\right)^{2}, \quad \beta=\left(\frac{\partial x}{\partial \xi}\right)\left(\frac{\partial x}{\partial \eta}\right)+\left(\frac{\partial y}{\partial \xi}\right)\left(\frac{\partial y}{\partial \eta}\right), \quad \gamma=\left(\frac{\partial x}{\partial \xi}\right)^{2}+\left(\frac{\partial y}{\partial \xi}\right)^{2}
$$

$S^{\Phi}(\xi, \eta)$ is the source term in $\xi, \eta$ coordinates, $\mathrm{J}$ is the Jacobian of transformation and is given by

$$
\mathrm{J}=\frac{\partial x}{\partial \xi} \frac{\partial y}{\partial \eta}-\frac{\partial x}{\partial \eta} \frac{\partial y}{\partial \xi}
$$

Now Eq.(2.10) is to be solved by satisfying the following boundary conditions in order to get the flow field around the hydrofoil.

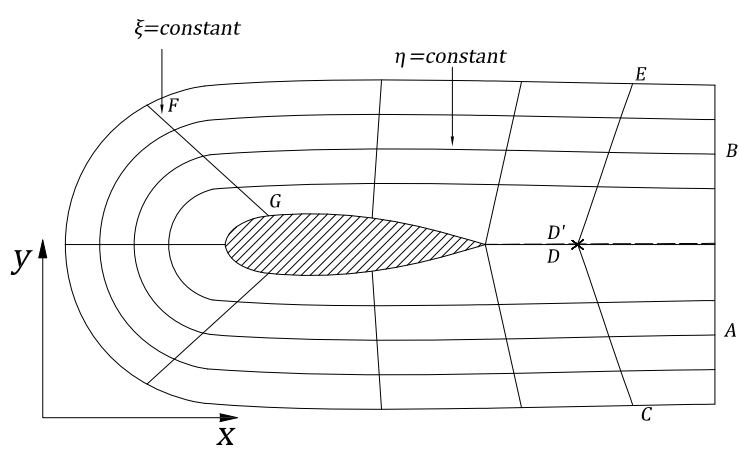

(a) Playsical domain

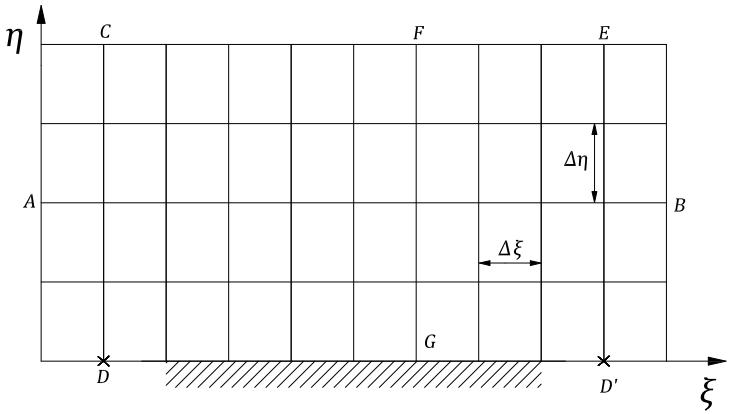

(b) Computational domain

Fig.1. Co-ordinate system and C-type control volume.

\subsection{Boundary conditions}

Neglecting the effect of free surface, the boundary conditions for a flow field around a hydrofoil fixed in a stream of uniform velocity $u_{0}$ can be written as:

(a) Inflow boundary: The components of the flow variables are provided as

$$
u=u_{0}, \quad v=0, \quad k=k_{0}, \quad \varepsilon=\varepsilon_{0} .
$$

(b) Outflow boundary: The outlet boundary is located far from the region of interest and the Reynolds number is high, the gradient in the flow direction is taken to be zero. Thus

$$
\frac{\partial u}{\partial n}, \frac{\partial v}{\partial n}, \frac{\partial k}{\partial n}, \frac{\partial \epsilon}{\partial n}=0
$$

where $\partial / \partial n$ is the derivative parallel to the streamlines;

(c) Solid boundary: The no slip boundary condition is applied on the surface of the hydrofoil.

$$
u=0, \quad v=0, \quad k=0, \quad \varepsilon=0 .
$$

(d) Far-field boundary: The flow variables on the symmetry plane are prescribed as

$$
u_{S}=u_{P}, \quad v=0, \quad k_{S}=k_{P}, \quad \varepsilon_{S}=\varepsilon_{P}
$$


Moreover, as the standard $k-\varepsilon$ turbulence model cannot be applied in the transition layer and also the viscous sublayer around the hydrofoil, the wall functions are to be adopted.

\section{The finite volume method}

\subsection{Discretization of governing equation in body fitted coordinates}

The discretization is performed following a finite control volume approach in which the computational domain is divided into a number of contiguous quadrilateral cells. A collocated grid arrangement is used in which all the variables are stored at the geometric center of the cell. The locations of the various dependent variables and the associated cells for this grid configuration are shown in Figs 2 ( $a$ and b). Equation (2.10) is integrated over the volume of each cell in the computational domain as

$$
\begin{aligned}
& \int_{C V}\left\{\frac{\partial(\rho U \Phi)}{\partial \xi}+\frac{\partial(\rho V \Phi)}{\partial \eta}\right\} d v= \\
= & \int_{C V}\left\{\frac{\partial}{\partial \xi}\left[\frac{\Gamma}{J}\left(\alpha \Phi_{\xi}-\beta \Phi_{\eta}\right)\right]+\frac{\partial}{\partial \eta}\left[\frac{\Gamma}{J}\left(\gamma \Phi_{\eta}-\beta \Phi_{\xi}\right)\right]+J S^{\Phi}(\xi, \eta)\right\} d v .
\end{aligned}
$$

Applying Gauss's divergence theorem to convert volume integrals to surface integrals, Eq.(3.1), after little rearrangement, may be written as

$$
\begin{aligned}
& (\rho U \Phi \Delta \eta)_{w}^{e}+(\rho V \Phi \Delta \xi)_{s}^{n}=\left(\frac{\Gamma}{J} \alpha \frac{\partial \Phi}{\partial \xi} \Delta \eta\right)_{w}^{e}+\left(\frac{\Gamma}{J} \gamma \frac{\partial \Phi}{\partial \eta} \Delta \xi\right)_{s}^{n}+ \\
& +\left\{-\left(\frac{\Gamma}{J} \beta \frac{\partial \Phi}{\partial \eta} \Delta \eta\right)_{w}^{e}-\left(\frac{\Gamma}{J} \beta \frac{\partial \Phi}{\partial \xi} \Delta \xi\right)_{s}^{n}+J\left(S_{c}+S_{P} \Phi_{P}\right) \Delta \xi \Delta \eta\right\} .
\end{aligned}
$$

The cross derivative terms have been added to the source term which in turn has been linearized as suggested by Patanker [13]. Using the notation of Fig.2, the following approximations may be made for the derivatives at face $e$ :

$$
\begin{aligned}
& \left(\frac{\partial x}{\partial \xi}\right)_{e} \approx \frac{x_{E}-x_{P}}{\xi_{E}-\xi_{P}}, \quad\left(\frac{\partial x}{\partial \eta}\right)_{e} \approx \frac{\left(x_{n}-x_{s}\right)_{e}}{\left(\eta_{n}-\eta_{s}\right)_{e}}=\frac{x_{n e}-x_{s e}}{\eta_{n e}-\eta_{s e}}, \\
& \left(\frac{\partial y}{\partial \xi}\right)_{e} \approx \frac{y_{E}-y_{P}}{\xi_{E}-\xi_{P}}, \quad\left(\frac{\partial y}{\partial \eta}\right)_{e} \approx \frac{\left(y_{n}-y_{s}\right)_{e}}{\left(\eta_{n}-\eta_{s}\right)_{e}}=\frac{y_{n e}-y_{s e}}{\eta_{n e}-\eta_{s e}} .
\end{aligned}
$$

Analogous expressions may be derived for other faces. Using Eqs (2.11), (2.12), (2.13) and Eq.(3.3), Eq.(3.2) can be written as

$$
\begin{aligned}
& {\left[F_{e} \Phi_{e}-F_{w} \Phi_{w}+F_{n} \Phi_{n}-F_{s} \Phi_{s}\right]=D_{e}\left(\Phi_{E}-\Phi_{P}\right)-D_{w}\left(\Phi_{P}-\Phi_{W}\right)+} \\
& +D_{n}\left(\Phi_{N}-\Phi_{P}\right)-D_{s}\left(\Phi_{P}-\Phi_{s}\right)+\left[N_{e}\left(\Phi_{n}-\Phi_{s}\right)_{e}-N_{w}\left(\Phi_{n}-\Phi_{s}\right)_{w}+\right. \\
& \left.+N_{n}\left(\Phi_{e}-\Phi_{w}\right)_{n}-N_{s}\left(\Phi_{e}-\Phi_{w}\right)_{s}+\left(S_{c}+S_{P} \Phi_{P}\right) \delta V\right] .
\end{aligned}
$$


Central differencing is used to discretize the diffusion terms and suitable interpolation for the convective terms is required to express cell face values in terms of nodal values. This is achieved from Demirdzic and Peric [14] by blending second-order central (CDS) differencing and first-order unconditionally stable upwind differencing scheme (UDS) in a deferred correction manner

$$
\left[\Phi_{e}^{U D S}\right]_{\text {implicit }}+\lambda\left[\Phi_{e}^{C D S}-\Phi_{e}^{U D S}\right]_{\text {explicit }}
$$

where $\lambda$ is the blending factor having value between 0 to 1 . The explicit part in Eq.(3.5) is obtained from previous iteration and added to the source term, like the cross derivative terms. Using the above scheme for convective terms and after little manipulation, Eq.(3.4) can be written in the following algebraic form

$$
a_{P} \Phi_{P}=a_{W} \Phi_{W}+a_{E} \Phi_{E}+a_{N} \Phi_{N}+a_{S} \Phi_{S}+S^{\prime} .
$$

Introducing an under-relaxation factor to slow down changes of the dependent variable in consecutive iterations, Eq.(3.6) becomes

$$
\begin{aligned}
& \left(\frac{a_{P}}{\alpha_{\Phi}}\right) \Phi_{P}=a_{W} \Phi_{W}+a_{E} \Phi_{E}+a_{N} \Phi_{N}+a_{S} \Phi_{S}+S_{\Phi} \\
& \left(\frac{a_{P}}{\alpha_{\Phi}}\right) \Phi_{P}=\sum_{n b} a_{n b} \Phi_{n b}+S_{\Phi}
\end{aligned}
$$

where

$$
\begin{aligned}
& \sum_{n b} a_{n b} \Phi_{n b}=a_{W} \Phi_{W}+a_{E} \Phi_{E}+a_{N} \Phi_{N}+a_{S} \Phi_{S} \\
& a_{P}=a_{W}+a_{E}+a_{N}+a_{S}-S_{P} \delta V+\left[F_{e}-F_{w}+F_{n}-F_{S}\right]=a_{E}+a_{N}+a_{S}-S_{P} \delta V .
\end{aligned}
$$

The terms inside the third bracket correspond to the continuity equation. After outer iteration steps, the mass fluxes are corrected so that the bracketed term vanishes identically and, therefore, are not considered.

$$
\begin{aligned}
& S_{\Phi}=S^{\prime}+\frac{\left(1-\alpha_{\Phi}\right)}{\alpha_{\Phi}} a_{P} \Phi_{P}^{m}= \\
& =N_{e}\left(\Phi_{n}-\Phi_{s}\right)_{e}-N_{w}\left(\Phi_{n}-\Phi_{s}\right)_{w}+N_{n}\left(\Phi_{e}-\Phi_{w}\right)_{n}-N_{s}\left(\Phi_{e}-\Phi_{w}\right)_{s}+ \\
& +\lambda\left\{\left[F_{e} \Phi_{e}-F_{w} \Phi_{w}+F_{n} \Phi_{n}-F_{S} \Phi_{s}\right]_{U D S}-\left[F_{e} \Phi_{e}-F_{w} \Phi_{w}+F_{n} \Phi_{n}-F_{S} \Phi_{s}\right]_{C D S}\right\}+ \\
& +S_{c} \delta V+\text { pterm }+\frac{\left(1-\alpha_{\Phi}\right)}{\alpha_{\Phi}} a_{P} \Phi_{P}^{m}, \\
& \text { pterm }\left\{\begin{array}{c}
=-\left(P_{e}-P_{w}\right)\left(y_{n}-y_{s}\right)+\left(P_{n}-P_{s}\right)\left(y_{e}-y_{w}\right) \text { for } x \text {-momentum } \\
=-\left(P_{n}-P_{s}\right)\left(x_{e}-x_{w}\right)+\left(P_{e}-P_{w}\right)\left(x_{n}-x_{s}\right) \text { for } y \text {-momentum } \\
=0 \quad \text { for all other equations }
\end{array}\right.
\end{aligned}
$$

\begin{tabular}{|c|c|c|c|}
\hline$a_{W}$ & $a_{E}$ & $a_{N}$ & $a_{S}$ \\
\hline & & $D_{n}+\max \left(0,-F_{n}\right)$ & $D_{s}+\max \left(F_{s}, 0\right)$ \\
$D_{w}+\max \left(F_{w}, 0\right)$ & $D_{e}+\max \left(0,-F_{e}\right)$ & $D^{\prime}$ & \\
\hline
\end{tabular}




$$
\begin{aligned}
D_{e}= & \frac{\Gamma\left\{\left(x_{n}-x_{s}\right)_{e}^{2}+\left(y_{n}-y_{s}\right)_{e}^{2}\right\}}{\left(x_{E}-x_{P}\right)\left(y_{n}-y_{s}\right)_{e}-\left(x_{n}-x_{s}\right)_{e}\left(y_{E}-y_{P}\right)}, \\
D_{w}= & \frac{\Gamma\left\{\left(x_{n}-x_{s}\right)_{w}^{2}+\left(y_{n}-y_{s}\right)_{w}^{2}\right\}}{\left(x_{P}-x_{W}\right)\left(y_{n}-y_{s}\right)_{w}-\left(x_{n}-x_{s}\right)_{w}\left(y_{P}-y_{W}\right)}, \\
D_{n}= & \frac{\Gamma\left\{\left(x_{e}-x_{w}\right)_{n}^{2}+\left(y_{e}-y_{w}\right)_{n}^{2}\right\}}{-\left(x_{N}-x_{P}\right)\left(y_{e}-y_{w}\right)_{n}+\left(x_{e}-x_{w}\right)_{n}\left(y_{N}-y_{P}\right)}, \\
D_{s}= & \frac{\Gamma\left\{\left(x_{e}-x_{w}\right)_{s}^{2}+\left(y_{e}-y_{w}\right)_{s}^{2}\right\}}{-\left(x_{P}-x_{S}\right)\left(y_{e}-y_{w}\right)_{s}+\left(x_{e}-x_{w}\right)_{s}\left(y_{P}-y_{S}\right)}, \\
F_{e}= & \rho\left\{u_{e}\left(y_{n}-y_{s}\right)_{e}-v_{e}\left(x_{n}-x_{s}\right)_{e}\right\}, F_{w}=\rho\left\{u_{w}\left(y_{n}-y_{s}\right)_{w}-v_{w}\left(x_{n}-x_{s}\right)_{w}\right\}, \\
F_{n}= & \rho\left\{-u_{n}\left(y_{e}-y_{w}\right)_{n}+v_{n}\left(x_{e}-x_{w}\right)_{n}\right\}, F_{s}=\rho\left\{-u_{s}\left(y_{e}-y_{w}\right)_{s}+v_{s}\left(x_{e}-x_{w}\right)_{s}\right\}, \\
N_{n}= & \frac{\Gamma\left\{\left(x_{N}-x_{P}\right)\left(x_{e}-x_{w}\right)_{n}+\left(y_{N}-y_{P}\right)\left(y_{e}-y_{w}\right)_{n}\right\}}{\left(x_{N}-x_{P}\right)\left(y_{e}-y_{w}\right)_{n}-\left(x_{e}-x_{w}\right)_{n}\left(y_{N}-y_{P}\right)}, \\
N_{e}= & \frac{\Gamma\left\{\left(x_{E}-x_{P}\right)\left(x_{n}-x_{s}\right)_{e}+\left(y_{E}-y_{P}\right)\left(y_{n}-y_{s}\right)_{e}\right\}}{\left(x_{n}-x_{s}\right)_{e}\left(y_{E}-y_{P}\right)-\left(x_{E}-x_{P}\right)\left(y_{n}-y_{s}\right)_{e}}, \\
& \Gamma\left\{\left(x_{P}-x_{W}\right)\left(x_{n}-x_{s}-x_{w}\right)_{s}+\left(y_{P}-y_{W}\right)\left(y_{n}-y_{s}\right)_{w}\right\}
\end{aligned},
$$

and the volume (area in 2D) of the cell $\delta V$ around the node $P$ as indicated in Fig.2a is

$$
\begin{aligned}
& \delta V=\left[\left(x_{e}-x_{w}\right)\left(y_{n}-y_{s}\right)-\left(x_{n}-x_{s}\right)\left(y_{e}-y_{w}\right)\right]= \\
& =\frac{1}{2}\left[\left(x_{n e}-x_{s w}\right)\left(y_{n w}-y_{s e}\right)-\left(y_{n e}-y_{s w}\right)\left(x_{n w}-x_{s e}\right)\right]
\end{aligned}
$$

$\Phi_{P}^{m}$ denotes the value of the dependent variable from the previous iteration and $\alpha_{\Phi}$ is the under relaxation factor. 


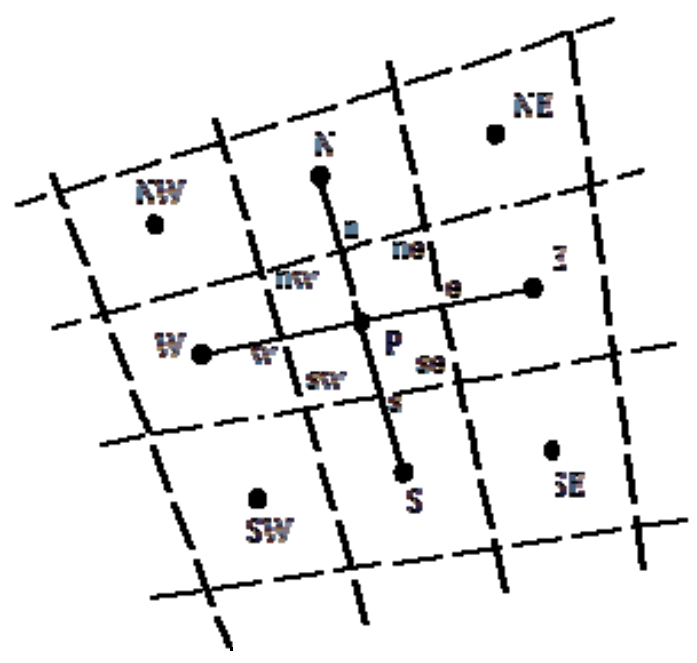

(a) Physical plane

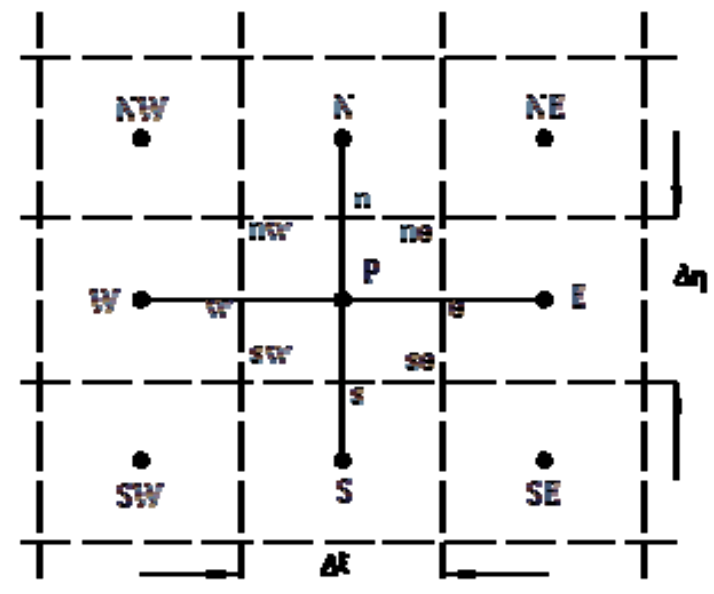

(b) Computational plane

Fig.2. Collocated grid arrangement.

\subsection{The wall functions}

To close the statement of the problem, we still need to prescribe the tangential stress as well as the boundary conditions for $\mathrm{k}$ and $\varepsilon$ on the solid boundary. Note that the equations of the $k-\varepsilon$ model are invalid in the vicinity of the wall where the Reynolds number is rather low and viscous effects are dominant. In the case of laminar flow, the no-slip wall boundary condition is directly applied at the wall. For turbulent flows however, to avoid using finer grids near walls, where steep cross flow gradients exist, 'wall functions' are used. These wall functions are given as follows

$$
\tau_{w}=\frac{\rho v_{t} C_{\mu}{ }^{\frac{1}{4}} k_{P}^{\frac{1}{2}}}{\frac{1}{\kappa} \ln \left(E n_{P}^{+}\right)}
$$

where

$$
n_{P}^{+}=\frac{\rho C_{\mu}{ }_{\mu}^{\frac{1}{4}} k_{P}^{\frac{1}{2}} n_{P}}{\mu},
$$

$v_{t}$ is the wall parallel velocity and $n_{P}$ is the distance of the first computational node normal to the wall.

$$
\begin{gathered}
G_{P}=\tau_{w} \frac{C_{\mu}^{\frac{1}{4}} k_{p}{ }^{\frac{1}{2}}}{\kappa n_{p}}, \\
\varepsilon_{P}=\frac{C_{\mu}{ }^{\frac{3}{4}} k_{p}{ }^{\frac{3}{2}}}{\kappa n_{p}} .
\end{gathered}
$$

\section{Solution by SIMPLE algorithm}

To obtain velocity and pressure fields and values of turbulent quantities, an iterative solution procedure akin to the SIMPLE method (Patankar and Spalding [16]) is used. In the present work the scalar 
and vector variables are stored in a collocated manner. Using Eq.(3.8), the momentum equations in the $x$ and $y$ directions may be written as

$$
\begin{aligned}
& \left(\frac{a_{P}}{\alpha_{u}}\right) u_{P}=\sum a_{n b} u_{n b}+S_{u}-\left(P_{e}-P_{w}\right)\left(y_{n}-y_{s}\right)+\left(P_{n}-P_{s}\right)\left(y_{e}-y_{w}\right), \\
& \left(\frac{a_{P}}{\alpha_{v}}\right) v_{P}=\sum a_{n b} u_{n b}+S_{v}+\left(P_{e}-P_{w}\right)\left(x_{n}-x_{s}\right)-\left(P_{n}-P_{s}\right)\left(x_{e}-x_{w}\right) .
\end{aligned}
$$

The pressure terms have been removed from the source terms in the above equations for convenience. Equations (4.1) and (4.2) may be written in the following matrix form.

$$
\begin{aligned}
& {\left[\boldsymbol{A}_{\boldsymbol{u}}\right]\{\boldsymbol{u}\}=\left\{\boldsymbol{S}_{\boldsymbol{u}}\right\},} \\
& {\left[\boldsymbol{A}_{\boldsymbol{v}}\right]\{\boldsymbol{v}\}=\left\{\boldsymbol{S}_{\boldsymbol{v}}\right\}}
\end{aligned}
$$

where $\{\boldsymbol{u}\},\{\boldsymbol{v}\}$ denotes the field of the unknown nodal velocity field arranged in vector form, $\{\boldsymbol{S}\}$ is a similar column vector containing source terms and $[\boldsymbol{A}]$ is the coefficient matrix. For the $(m+l)^{\text {th }}$ outer iteration, the matrix $\left[\boldsymbol{A}_{\boldsymbol{u}}\right]$ and column vector $\boldsymbol{S}_{\boldsymbol{u}}$ are obtained using the tentative values of the parameters $u^{m}, v^{m}, k^{m}, \varepsilon^{m}, P^{m}$. These parameters superscripted by $m$ are either initial guesses or solution of the governing equations at the $m^{\text {th }}$ outer iteration. Once matrix $\left[\boldsymbol{A}_{\boldsymbol{u}}\right]$ and column vector $\boldsymbol{S}_{\boldsymbol{u}}$ are assembled, the system of equations represented by Eq.(4.3) is solved within the inner iteration loop by the strongly implicit procedure of Stone [17]. The velocity field $u^{*}$, obtained in this manner satisfies the following equation

$$
\left[A_{u}\right]\left\{u^{*}\right\}=\left\{S_{u}\right\}
$$

The asterisk is used to indicate that the computed velocity field satisfies momentum but not necessarily continuity equation. Similarly, $v^{*}$ satisfies the following system

$$
\left[A_{u}\right]\left\{v^{*}\right\}=\left\{S_{v}\right\}
$$

However, the success of the SIMPLE algorithm in case of collocated arrangement of variables depends on the interpolation of nodal velocities to obtain face velocities and hence mass fluxes. To avoid false pressure field special interpolation technique suggested by Rhie [1] is employed which leads to the following $u$ velocity at face $e$

$$
u_{e}^{*}=\alpha_{u}\left[\frac{\sum a_{n b} u_{n b}^{*}+B_{P}}{a_{p}}\right]_{e}-\alpha_{u} \overline{\left(\frac{1}{a_{P}}\right)_{e}}\left(y_{n}-y_{s}\right)_{e}\left(P_{E}^{m}-P_{P}^{m}\right)
$$

where $B_{P}$ is the source term excluding pressure gradient across the cell. The over bar denotes linear interpolation between two neighboring nodes which for an arbitrary quantity $g$ is given by 


$$
g=f_{e}^{+} g+\left(1-f_{e}^{+}\right) g
$$

where

$$
f_{e}^{+}=\frac{|\overline{P e}|}{\overline{\mid P e}|+\overline{\mid e E}|} .
$$

Similarly, velocities at other faces may be obtained in both $x$ and $y$ directions. The mass fluxes obtained from these face velocities are not guaranteed to satisfy the discrete continuity equation. That is

$$
\begin{aligned}
& F_{e}^{*}-{F_{w}}^{*}+{F_{n}}^{*}-{F_{s}}^{*} \neq 0, \\
& F_{e}{ }^{*}-{F_{w}}^{*}+{F_{n}}^{*}-{F_{s}}^{*}=S_{m} .
\end{aligned}
$$

Consequently, the face velocities are corrected in the spirit of the SIMPLE algorithm as follows

$$
\begin{aligned}
& u_{e} \approx u_{e}^{*}+u_{e}^{\prime}, \\
& u_{e} \approx u_{e}^{*}+\alpha_{u}\left(y_{e}-y_{w}\right)_{e} \overline{\left(\overline{\left.p_{n}^{\prime}-p_{s}^{\prime}\right)_{e}}\right.}-\alpha_{u}\left(y_{n}-y_{s}\right)_{e} \overline{\left(\frac{1}{\left(a_{P}\right)_{e}}\right)_{P}}\left(p_{e}^{\prime}-p_{P}^{\prime}\right), \\
& v_{e} \approx v_{e}^{*}+v_{e}^{\prime}, \\
& v_{e} \approx v_{e}^{*}-\alpha_{v}\left(x_{e}-x_{w}\right)_{e} \frac{\overline{\left(p_{n}^{\prime}-p_{s}^{\prime}\right)_{e}}}{\left(a_{P}\right)_{e}}+\alpha_{v}\left(x_{n}-x_{s}\right)_{e} \overline{\left(\frac{1}{a_{P}}\right)_{e}}\left(p_{E}^{\prime}-p_{P}^{\prime}\right) .
\end{aligned}
$$

Using Eqs (4.11) and (4.12) the mass flux at face $e$ is found to be

$$
\begin{aligned}
& F_{e}=\rho\left\{-\alpha_{u} \overline{\left(\frac{1}{a_{P}}\right)_{e}}\left(y_{n}-y_{s}\right)_{e}^{2}\left(p_{E}^{\prime}-p_{P}^{\prime}\right)-\alpha_{v} \overline{\left(\frac{1}{a_{P}}\right)_{e}}\left(x_{n}-x_{s}\right)_{e}^{2}\left(p_{E}^{\prime}-p_{P}^{\prime}\right)\right\}+ \\
& +\rho\left\{\alpha_{u}\left(y_{e}-y_{w}\right)_{e}\left(y_{n}-y_{s}\right)_{e} \frac{\overline{\left(p_{n}^{\prime}-p_{s}^{\prime}\right)_{e}}}{\left(a_{P}\right)_{e}}+\alpha_{v}\left(x_{n}-x_{s}\right)_{e}\left(x_{e}-x_{w}\right)_{e} \frac{\overline{\left(p_{n}^{\prime}-p_{s}^{\prime}\right)_{e}}}{\left(a_{P}\right)_{e}}\right\}+ \\
& +\rho\left[u_{e}^{*}\left(y_{n}-y_{s}\right)_{e}-v_{e}^{*}\left(x_{n}-x_{s}\right)_{e}\right] .
\end{aligned}
$$

Neglecting the second term (cross-diffusion term) of Eq.(4.13) based on the recommendation of Peric [15] we get

$$
\begin{aligned}
& F_{e}=-\rho\left\{\alpha_{u} \overline{\left(\frac{1}{a_{P}}\right)_{e}}\left(y_{n}-y_{s}\right)_{e}^{2}+\alpha_{v} \overline{\left(\frac{1}{a_{P}}\right)_{e}}\left(x_{n}-x_{s}\right)_{e}^{2}\right\}\left(p_{E}^{\prime}-p_{P}^{\prime}\right)+ \\
& +\rho\left[u^{*}{ }_{e}\left(y_{n}-y_{s}\right)_{e}-v_{e}^{*}\left(x_{n}-x_{s}\right)_{e}\right] .
\end{aligned}
$$


Similarly, we can write for other faces

$$
\begin{aligned}
& F_{w}=-\rho\left\{\alpha_{u} \overline{\left(\frac{1}{a_{P}}\right)_{w}}\left(y_{n}-y_{s}\right)_{w}^{2}+\alpha_{v} \overline{\left(\frac{1}{a_{P}}\right)_{w}}\left(x_{n}-x_{s}\right)_{w}^{2}\right\}\left(p_{P}^{\prime}-p_{W}^{\prime}\right)+ \\
& +\rho\left[u_{w}^{*}\left(y_{n}-y_{s}\right)_{w}-v_{w}^{*}\left(x_{n}-x_{s}\right)_{w}\right], \\
& F_{n}=-\rho\left\{\alpha_{u} \overline{\left(\frac{1}{a_{P}}\right)_{n}}\left(y_{e}-y_{w}\right)_{n}^{2}+\alpha_{v} \overline{\left(\frac{1}{a_{P}}\right)_{n}}\left(x_{e}-x_{w}\right)_{n}^{2}\right\}\left(p_{N}^{\prime}-p_{P}^{\prime}\right)+ \\
& +\rho\left[-u_{n}^{*}\left(y_{e}-y_{w}\right)_{n}+v_{n}^{*}\left(x_{e}-x_{w}\right)_{n}\right], \\
& F_{S}=-\rho\left\{\alpha_{u} \overline{\left(\frac{1}{a_{P}}\right)_{S}}\left(y_{e}-y_{w}\right)_{s}^{2}+\alpha_{v} \overline{\left(\frac{1}{a_{P}}\right)_{s}}\left(x_{e}-x_{w}\right)_{s}^{2}\right\}\left(p_{P}^{\prime}-p_{S}^{\prime}\right)+ \\
& +\rho\left[-u_{s}^{*}\left(y_{e}-y_{w}\right)_{s}+v_{s}^{*}\left(x_{e}-x_{w}\right)_{s}\right] .
\end{aligned}
$$

Substituting Eq.(4.14) to Eq.(4.17) in discretized continuity Eq.(4.18) for incompressible flow the pressure correction Eq.(4.19) is derived after algebraic manipulation

$$
\begin{aligned}
& F_{e}-F_{w}+F_{n}-F_{S}=0, \\
& a_{P} p_{P}^{\prime}=a_{W} p_{W}^{\prime}+a_{E} p_{E}^{\prime}+a_{N} p_{S}^{\prime}+a_{S} p_{N}^{\prime}+S_{m}
\end{aligned}
$$

where

$$
\begin{aligned}
& a_{P}=a_{W}+a_{E}+a_{N}+a_{S}, \\
& a_{E}=\rho\left\{\alpha_{u} \overline{\left(\frac{1}{a_{P}}\right)_{e}}\left(y_{n}-y_{s}\right)_{e}^{2}+\alpha_{v} \overline{\left(\frac{1}{a_{P}}\right)_{e}}\left(x_{n}-x_{s}\right)_{e}^{2}\right\}, \\
& a_{W}=\rho\left\{\alpha_{u} \overline{\left(\frac{1}{a_{P}}\right)_{w}}\left(y_{n}-y_{s}\right)_{w}^{2}+\alpha_{v} \overline{\left(\frac{1}{a_{P}}\right)}\left(x_{w}-x_{s}\right)_{w}^{2}\right\}, \\
& a_{N}=\rho\left\{\alpha_{u}\left(\overline{\left.\frac{1}{a_{P}}\right)_{n}}\left(y_{e}-y_{w}\right)_{n}^{2}+\alpha_{v} \overline{\left(\frac{1}{a_{P}}\right)_{n}}\left(x_{e}-x_{w}\right)_{n}^{2}\right\},\right. \\
& a_{S}=\rho\left\{\alpha_{u}\left(\overline{\left.\frac{1}{a_{P}}\right)_{s}}\left(y_{e}-y_{w}\right)_{s}^{2}+\alpha_{v}\left(\frac{1}{a_{P}}\right)_{s}\left(x_{e}-x_{w}\right)_{s}^{2}\right\},\right. \\
& S_{m}=\left[\left[u_{w}^{*}\left(y_{n}-y_{s}\right)_{w}-v_{w}^{*}\left(x_{n}-x_{s}\right)_{w}\right]\right]-\left[u_{e}^{*}\left(y_{n}-y_{s}\right)_{e}-v_{e}^{*}\left(x_{n}-x_{s}\right)_{e}\right]+ \\
& +\left[-u_{S}^{*}\left(y_{e}-y_{w}\right)_{s}+v_{s}^{*}\left(x_{e}-x_{w}\right)_{s}\right]-\left[-u_{n}^{*}\left(y_{e}-y_{w}\right)_{n}+v_{n}^{*}\left(x_{e}-x_{w}\right)_{n}\right] .
\end{aligned}
$$


Pressure corrections are obtained by solving Eq.(4.19) by ILU decomposition method of Stone [17]. These pressure corrections may be in turn used to correct mass fluxes, nodal velocities and pressure as follows

$$
\begin{aligned}
& F_{e}^{m+1}=u_{e}^{*}\left(y_{n}-y_{s}\right)_{e}-v_{e}^{*}\left(x_{n}-x_{s}\right)_{e}+ \\
& -\rho\left\{\alpha_{u} \overline{\left(\frac{1}{a_{P}}\right)_{e}}\left(y_{n}-y_{s}\right)_{e}^{2}+\alpha_{v}\left(\frac{1}{a_{P}}\right)_{e}\left(x_{n}-x_{s}\right)_{e}^{2}\right\}\left(p_{E}^{\prime}-p_{P}^{\prime}\right), \\
& u_{P}^{m+1}=u_{P}^{*}-\alpha_{u}\left(y_{n}-y_{s}\right)_{e} \overline{\left(\frac{1}{a_{P}}\right)_{e}}\left(p_{E}^{\prime}-p_{P}^{\prime}\right), \\
& P^{m+1}=P^{m}+\alpha_{p} p^{\prime} .
\end{aligned}
$$

Since the flow is turbulent, the system of Eqs (4.23) and (4.24) for $k$ and $\varepsilon$ are solved next by strongly implicit procedure of Stone [17] to yield the quantities $k^{m+1}$ and $\varepsilon^{m+1}$ which mark the completion of the $(m+l)^{\text {th }}$ outer iteration

$$
\begin{aligned}
& {\left[A_{k}\right][k]=\left[S_{k}\right],} \\
& {\left[A_{\varepsilon}\right][\varepsilon]=\left[S_{\varepsilon}\right] .}
\end{aligned}
$$

The obtained flow variables $u^{m+1}, v^{m+1}, P^{m+1}, k^{m+1}, \varepsilon^{m+1}$ act as 'initial guesses' for the $(m+2)^{t h}$ outer iteration and the whole process described above is repeated until the convergence criterion is met.

\section{Convergence criteria}

Starting from initial guess for all field values the process of solving the equations is repeated until convergence. Due to coupling of variables and the nonlinearity of the equations, it is not necessary to solve exactly the discretized equations for a given set of coefficients (inner iteration); these are only approximate and need to be updated. So, inner iterations of momentum equations and equations of turbulent quantities are terminated by limiting the number of iteration to 1 . Convergence of the pressure correction equation is monitored by comparing the sum of the absolute residuals after each sweep to its initial value.

For outer iterations (solution with updated coefficients), the sum of the absolute values of the residuals over all control volumes is calculated, and normalized by inlet flux of the relevant quantity, $f_{\varphi}^{\text {inlet }}$. That is

$$
\bar{R}_{\varphi}=\frac{\sum_{l=1}^{K} R_{\varphi}^{l}}{f_{\varphi}^{\text {inlet }}} .
$$

For convergence of outer iteration to take place the following must be satisfied

$$
\max \left(\bar{R}_{u}, \bar{R}_{v}, \bar{R}_{l}, \bar{R}_{k}, \bar{R}_{\varepsilon}\right) \leq \psi .
$$


The above criterion ensures that the relative changes in the variables from one iteration to the next are of the order of $\psi$ or less.

\section{Results and discussion}

The turbulent flow over NACA 0012 and NACA 4412 hydrofoil forms a fascinating amalgam of fluid mechanics phenomena and its analysis has been carried out in the present work.

\subsection{NACA 0012 hydrofoil}

The flow past an NACA 0012 hydrofoil with $2.5 \mathrm{~m}$ chord length $(C)$ is simulated at $R e_{c}=2.8 \times 10^{6}$ with grid sizes of $50 \times 14,88 \times 20,176 \times 40$ and $0,6,10$ degrees of incidence. A typical grid arrangement for $176 \times 40$ cells is shown in Fig.3. In the present simulation the inlet boundary at the front of the foil along with the upper and bottom boundaries are located 4 chord lengths away from the leading edge. The outlet boundary at the rear side is 8 chord lengths away from the leading edge. The under-relaxation factors used are $\alpha_{u}=\alpha_{v}=\alpha_{k}=\alpha_{\varepsilon}=0.7, \alpha_{p}=0.1$ and the value of blending factor is $\lambda=0.5$.

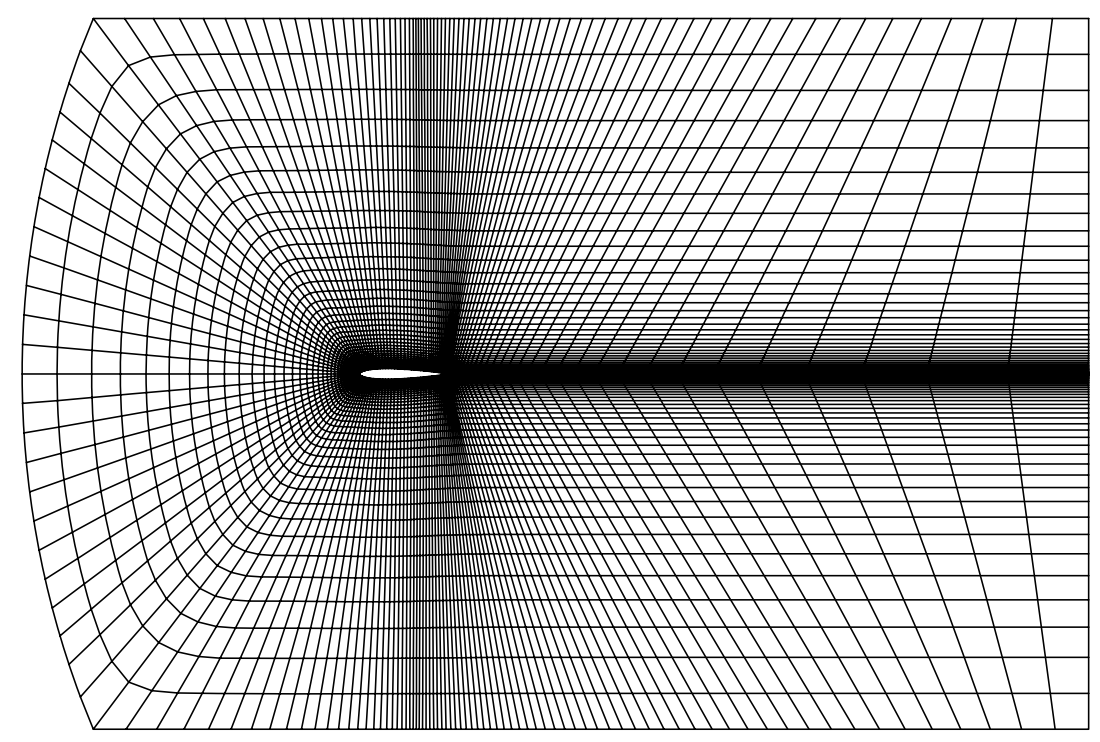

Fig.3. Grid arrangement for $176 \times 40$ cells.

A comparison of obtained pressure coefficients with its experimental results for each angle and a grid size of $176 \times 40$ is shown in Figs $4 \mathrm{a}, \mathrm{b}$, c. From these figures it is evident that the computed results agree very well with the experiment. In fact, at 0 and 6-degree incidence the result agrees better than that obtained by Rhie [1] near the leading edge. However, discrepancy can be noted at the leading and trailing edges which may be attributed partly to inadequate turbulence modeling. Moreover, the mesh may not be sufficiently fine to capture the very strong gradients existing in those regions. In addition to the pressure coefficient curves, the pressure contours and streamlines at 6 degrees incidence are shown in Figs 5 and 6 for a grid size of $176 \times 40$ at $R e_{c}=2.8 \times 10^{6}$. 


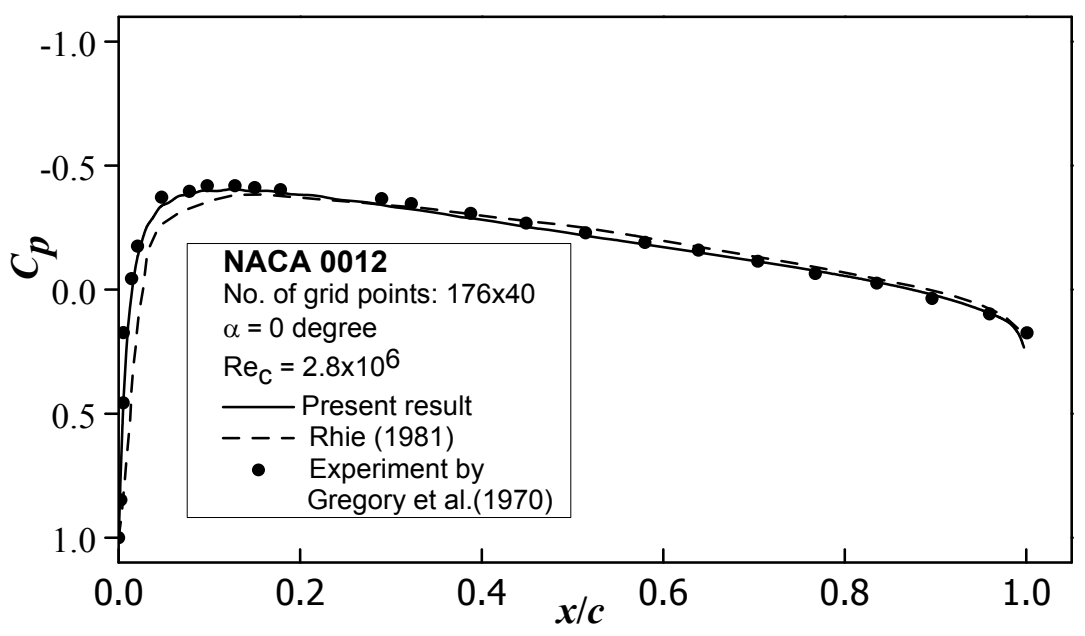

(a) Angle of attack 0 degree

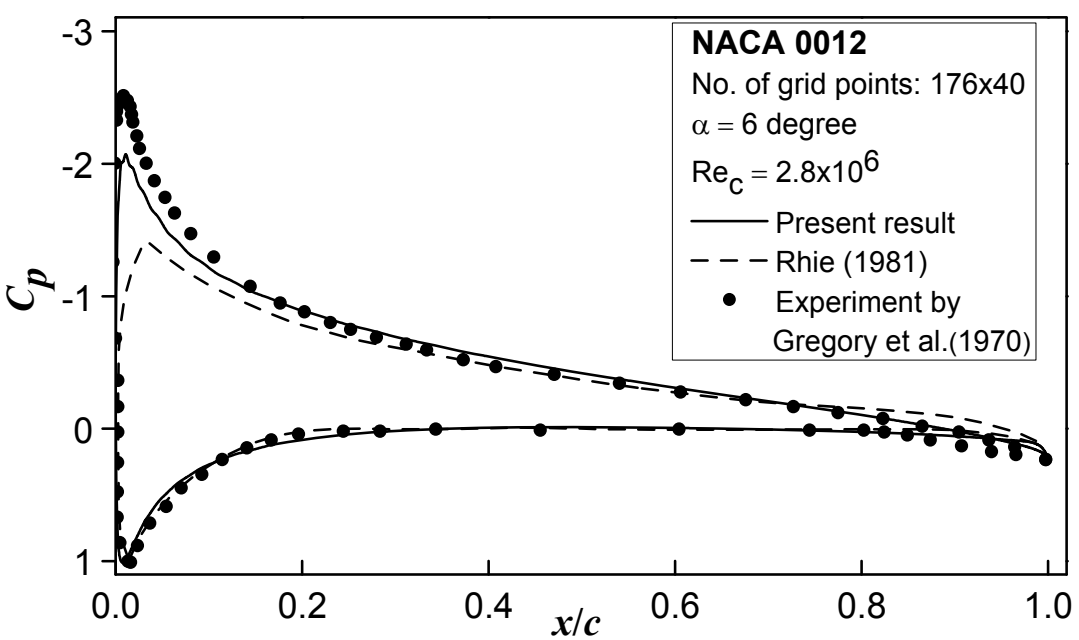

(b) Angle of attack 6 degree

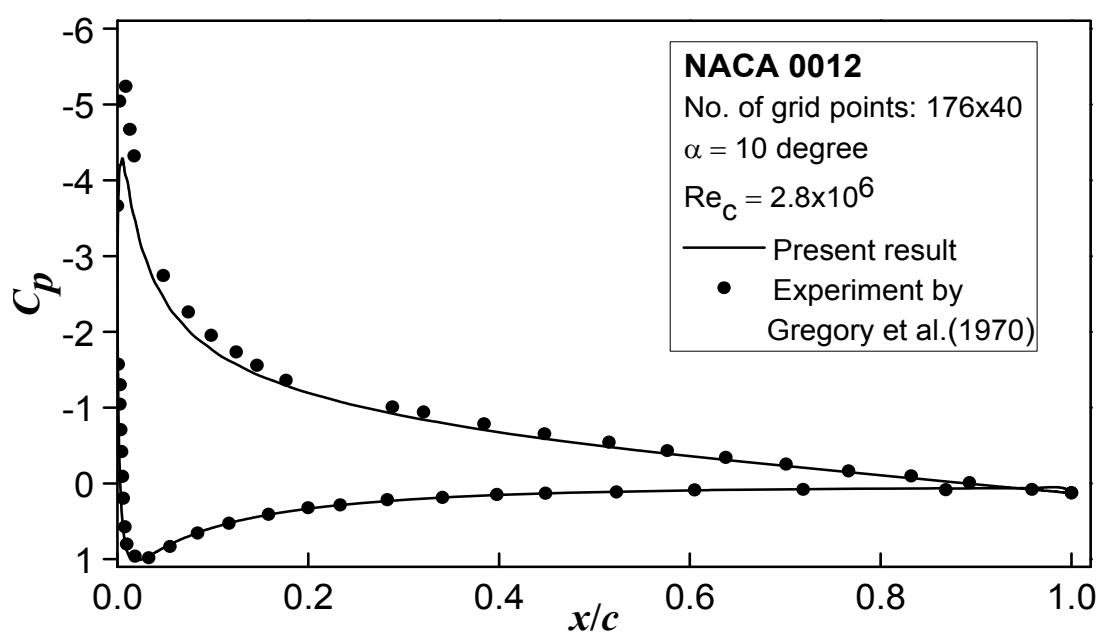

(c) Angle of attack 10 degree

Fig.4. Surface pressure distribution for NACA 0012 hydrofoil at (a) 0 degree (b) 6 degree and (c) 10 degree angle of incidence. 


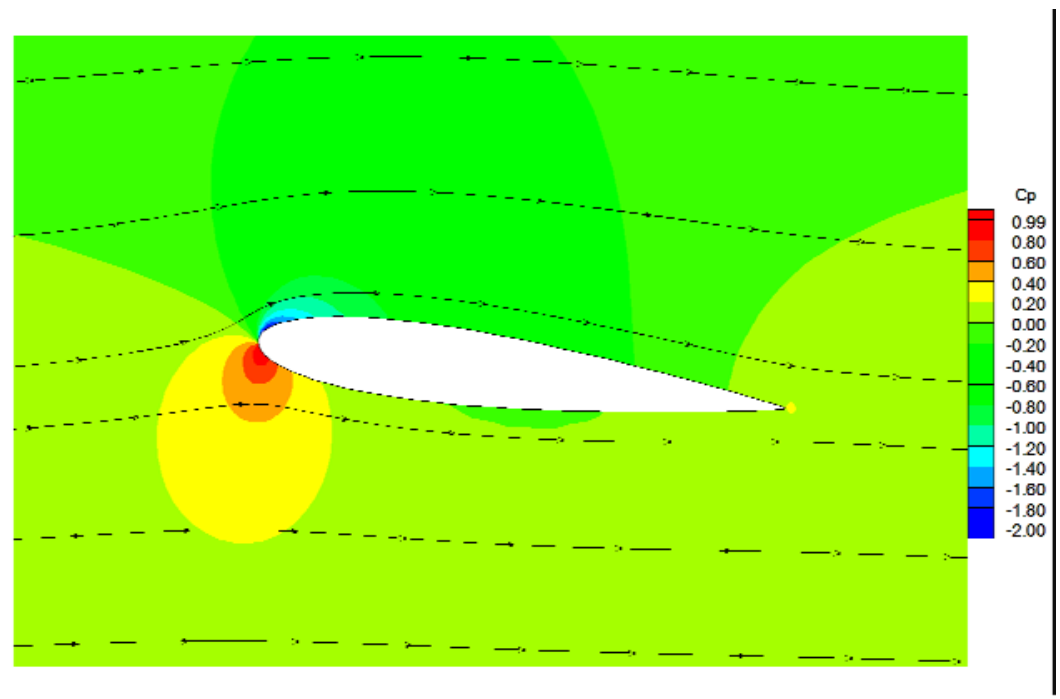

Fig.5. Pressure coefficient contours for NACA 0012 hydrofoil at 6 degree incidence.

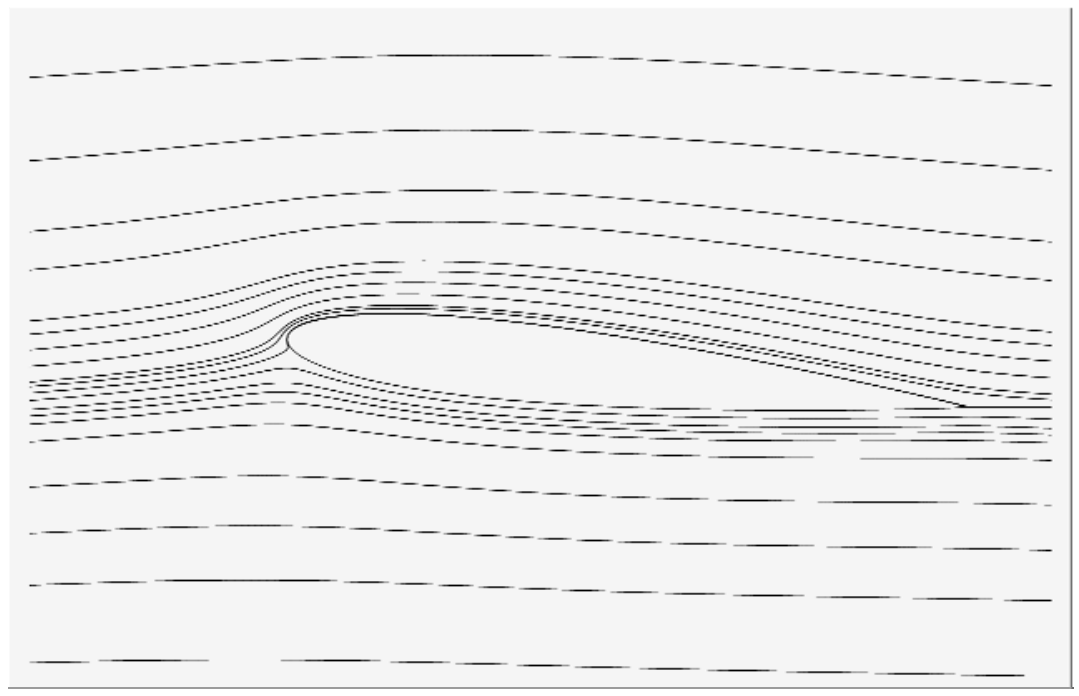

Fig.6. Streamlines for NACA 0012 hydrofoil at 6 degree incidence.

The $\mathrm{C}$ type structured mesh with three different sizes was isused to investigate the influence on surface pressure coefficients. At 10 degree angle of attack, pressure coefficients are computed at each of the three grids and plotted in Fig.7. It is evident that refinement of the grid gives increased numerical accuracy of pressure coefficients in the present case. Significant improvement is found on the suction side of the hydrofoil. However, the effect on the pressure side (where the discrepancy is small) is less considerable.

The improvement in result is particularly marked at the point of the lowest pressure coefficient near the leading edge. The percentage errors compared to experimental findings at this point for $50 \times 14,88 \times 20$ and $176 \times 40$ are found to be $50.0 \%, 33.0 \%$ and $16.6 \%$, respectively. There is a reduction in discrepancy because the steep gradients could be better resolved by the finer meshes. It may be also observed that improvement in regions other than the leading edge seems to be less marked when the grid size is increased from $88 \times 20$ to $176 \times 40$. So at a grid size of $176 \times 40$, the solution tends to become grid independent except at the leading edge. The slight error incurred in these regions for the most refined grid may therefore be attributed to 
inadequate turbulence modeling. The effect of angle of attack for $176 \times 40$ grid is also studied by plotting the pressure coefficients at 0,6 and 10 degree on the same axes as shown in Fig.8.

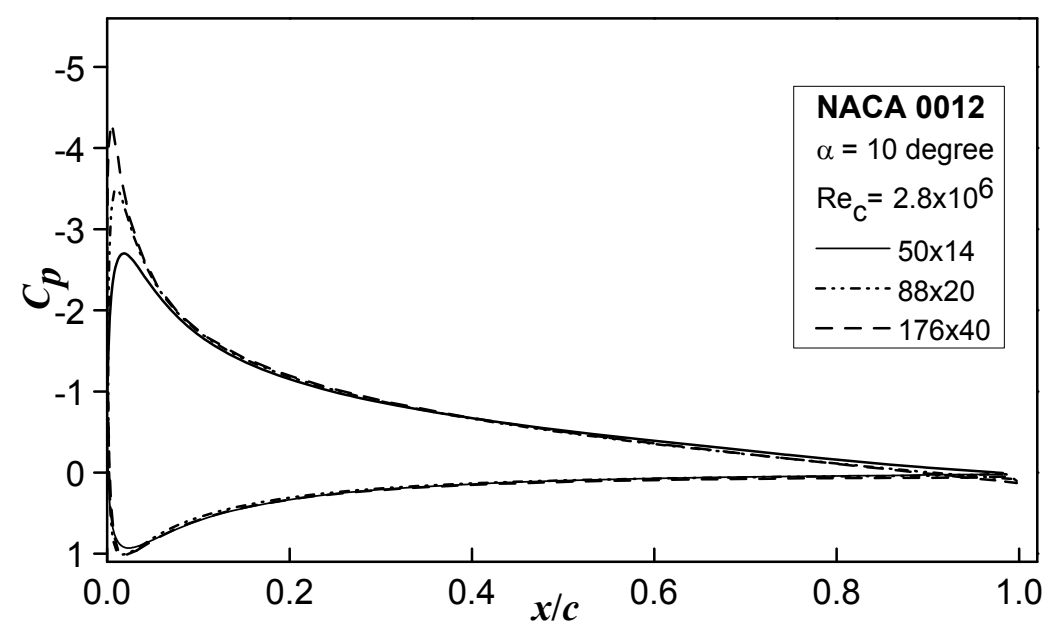

Fig.7. Grid dependency for NACA 0012 hydrofoil at 10 degree angle of incidence.

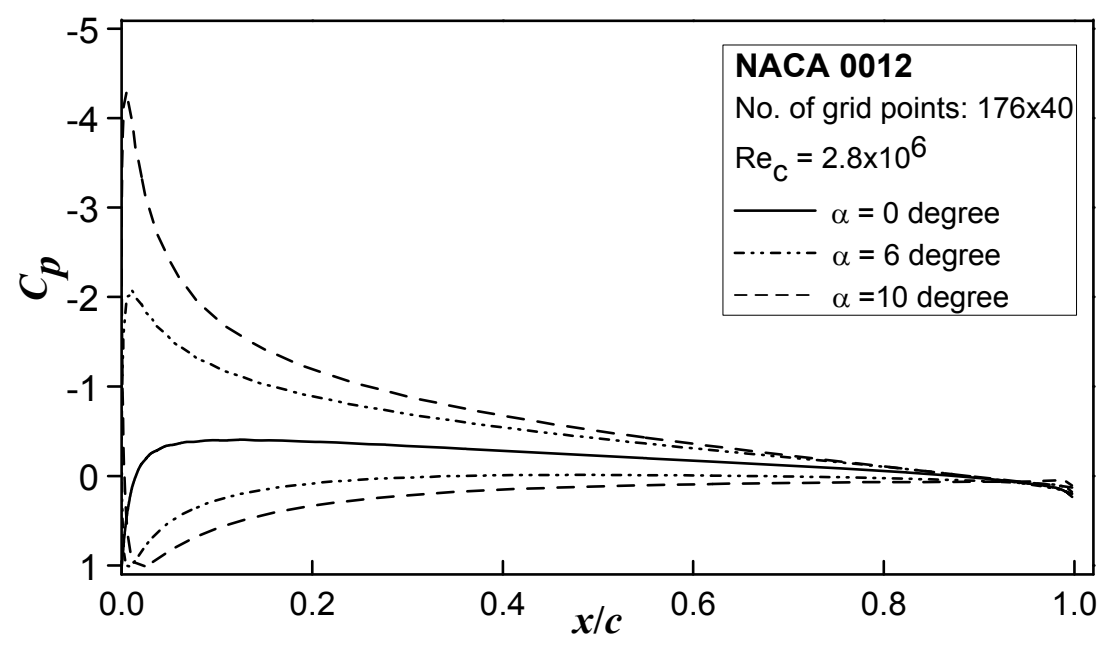

Fig.8. Effect of angle of attack on the pressure distribution for NACA 0012 hydrofoil.

\subsection{NACA 4412 hydrofoil}

Turbulent flow past NACA 4412 hydrofoil with $2.5 \mathrm{~m}$ chord length $(\mathrm{C})$ is analyzed using a method similar to that of NACA 0012. In this case the first mesh consists of 76 cells in the $\xi$ direction and 20 in the $\eta$ direction $(76 \times 20)$ with 1520 cells in total. The subsequent finer meshes have sizes of $(134 \times 25)$ and $(268 \times 50)$ with 3350 and 13400 cells, respectively.

The pressure coefficients are computed at $R e_{c}=3.1 \times 10^{6}$ and compared with Pinkerton [18] for a $268 \times 50 \mathrm{C}$ type structured grid as shown in Figs 9a, b, c, d. Since Pinkerton's experiment is for three dimensional flows, comparisons are made at effective angles of attack. The effective angles of attack for 1.2 degree, 2.9 degree, 6.4 degree and 10 degree are obtained according to Pinkerton [18] by subtracting the theoretically calculated induced angle of attack from the geometric angle of incidence. From the curves it can be seen that, at all the angles of attack, the agreement on the pressure side is excellent with slight discrepancy 
on the trailing edge. Discrepancy is more notable on the suction side, particularly near the leading edge. For 2.9 and 6.4 degree angle of incidence, little discrepancy also exists between $x / c$ of 0.1 to 0.3 which is believed to occur partly due to error incurred in calculating the effective angle of attack by Pinkerton [18].

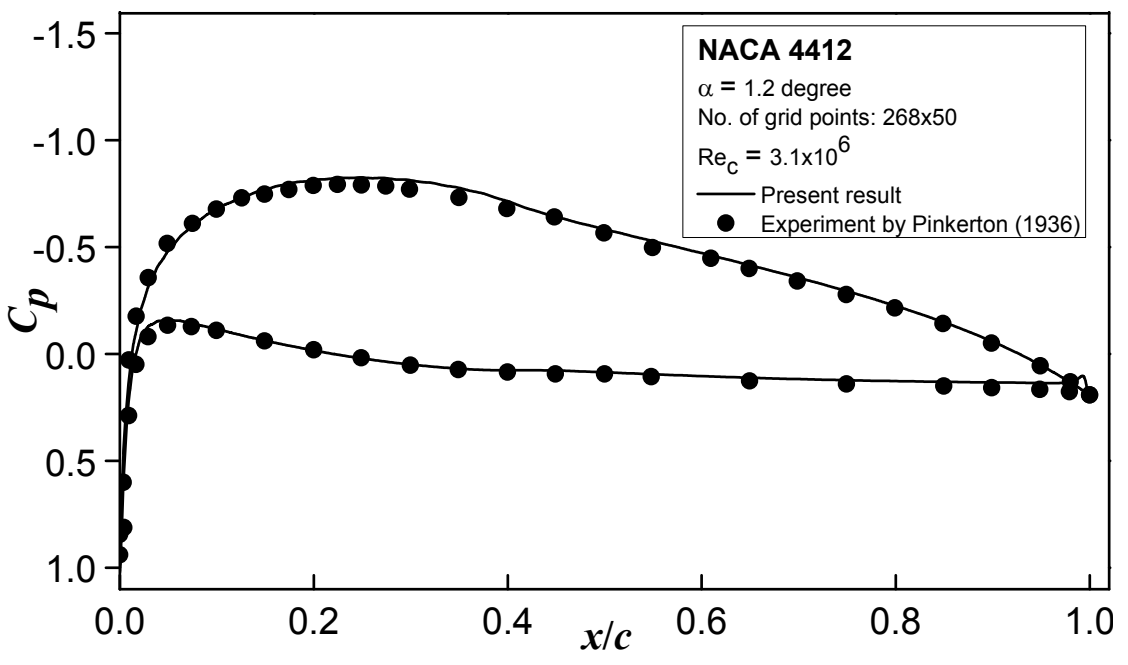

(a) Angle of attack 1.2 degree

(b)

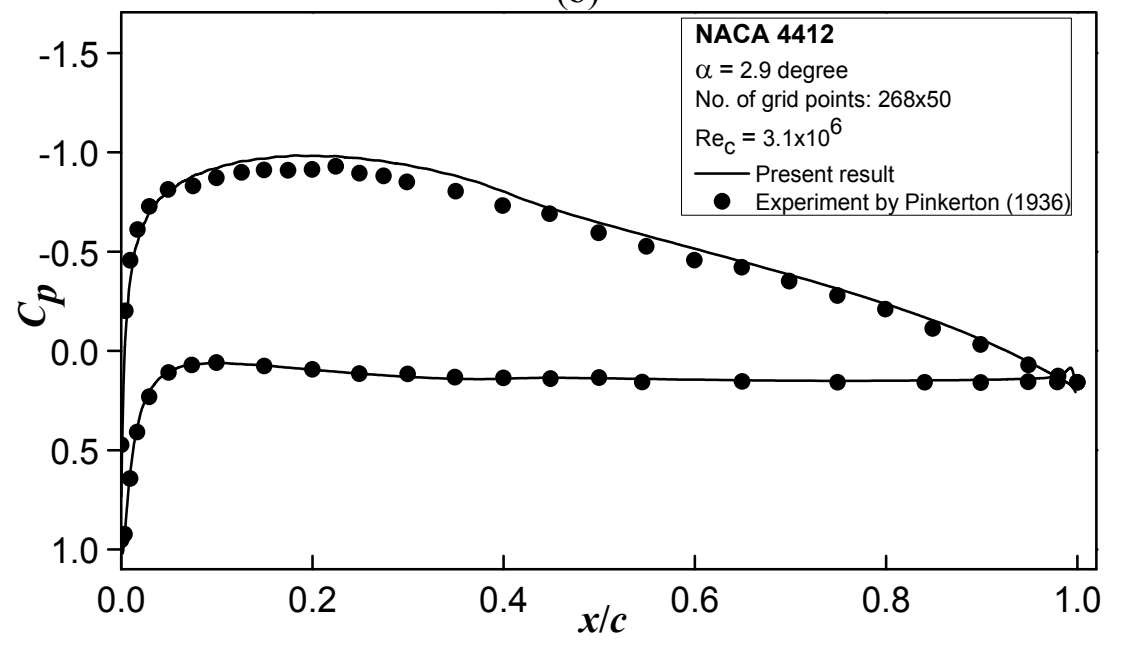

(c) Angle of attack 2.9 degree 


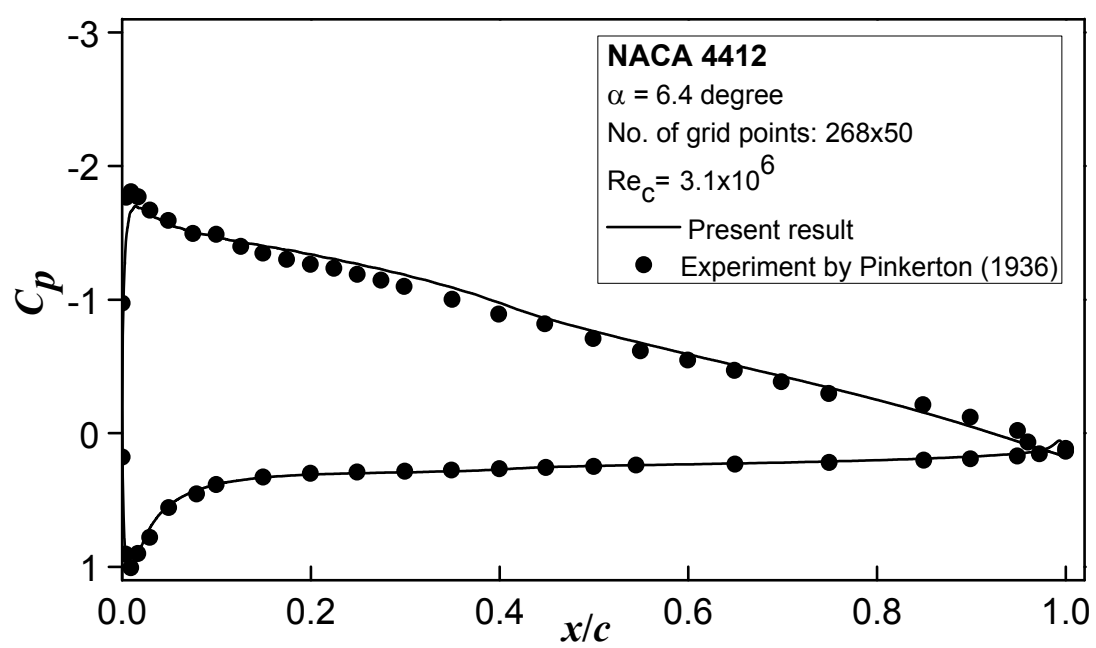

(d) Angle of attack 6.4 degree

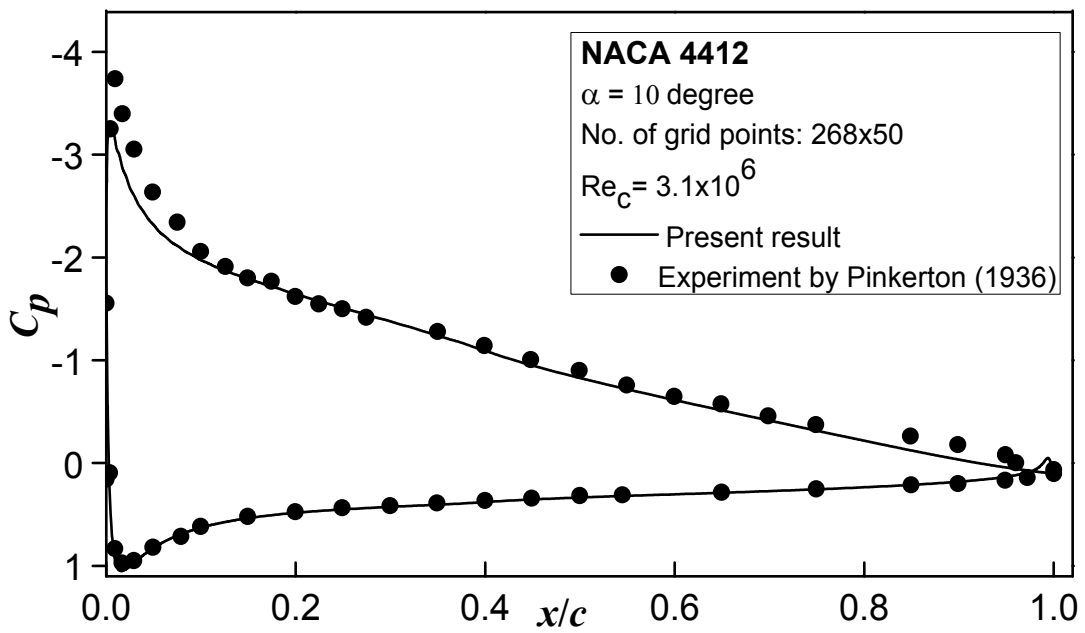

(d) Angle of attack 10 degree

Fig.9. Pressure coefficients for NACA 4412 hydrofoil for (a) 1.2 degree (b) 2.9 degree (c) 6.4 degree and (d) 10 degree angle of incidence.

The pressure coefficients at an angle of attack 10 degree are plotted in Fig.10 for three grid sizes on the same axes at $R e_{c}=3.1 \times 10^{6}$. It is evident that refinement of the grid gives increased numerical accuracy of pressure coefficients for NACA 4412 hydrofoil as well. However, except near the leading edge, the pressure coefficient values are seen to be affected very little when mesh size is increased from $134 \times 25$ to $268 \times 50$. The pressure coefficient values therefore exhibited grid independency when the finest mesh was employed. So, the very small amount of discrepancy extant with the most refined grid may have resulted due to inadequate modeling of turbulence and perhaps inaccuracy in the effective angle values. The effect of angle of attack for a mesh size of $268 \times 50$ is plotted in Fig.11. In addition, the lift coefficient is computed and validated in Fig.12 against the experimental results of Coles and Wadcock [19] and Kermeen [20]. Using a $268 \times 50$ grid size, flow over NACA 4412 hydrofoil is also simulated at $R e_{c}=3.1 \times 10^{6}$ at 13.9 degree incidence. The pressure coefficients on both suction and pressure side are computed and compared with Coles and Wadcock [19] as shown in Fig.13. Streamlines and velocity contours showing the separation near trailing edge are drawn in Figs 14 and 15 for 13.9 degree incidence. Despite the doubt in applicability of the 
$k-\varepsilon$ turbulence model in separated regions, the pressure coefficients agreed quite well. However, discrepancy in pressure coefficient values exists near the leading edge and trailing edge. Also the error on the suction side of the hydrofoil is more considerable than the pressure side and the flow separation occurs earlier than found in the experiment. The pressure computed on the suction side is less negative compared to Coles and Wadcock [19]. This may overestimate the adverse pressure gradient resulting in early flow separation. Figure 16 shows the convergence history for NACA 0012 and NACA 4412 hydrofoils at 10 degree incidence which illustrates the satisfactory convergence rate for both NACA 0012 and NACA 4412 despite the use of a simplified pressure correction equation.

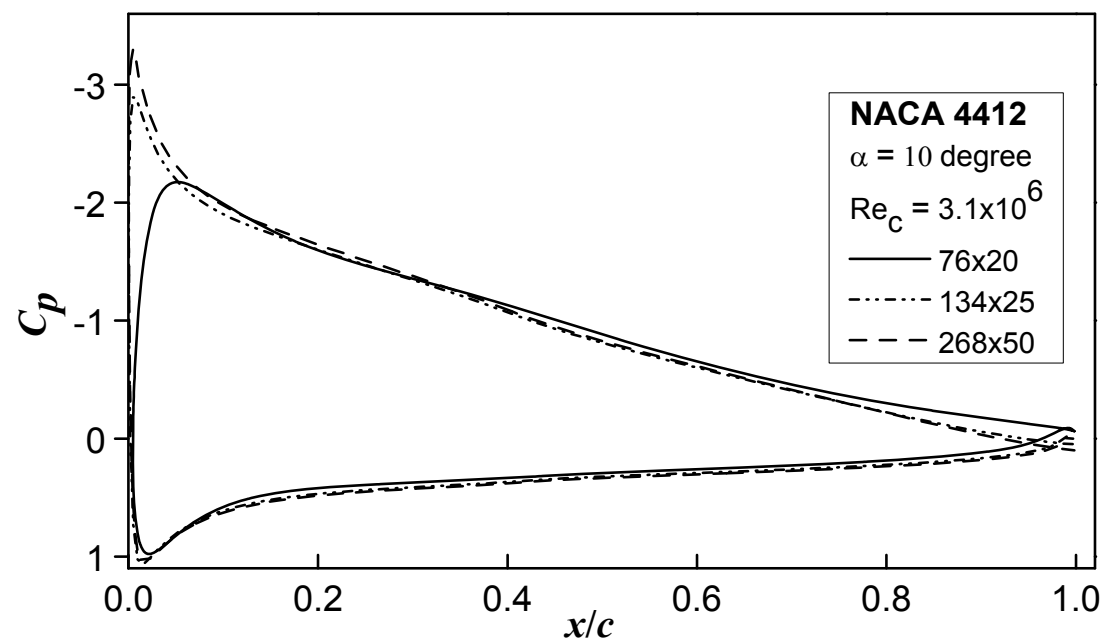

Fig.10. Grid dependency of pressure coefficients for NACA 4412 hydrofoil at 10 degree angle of incidence.

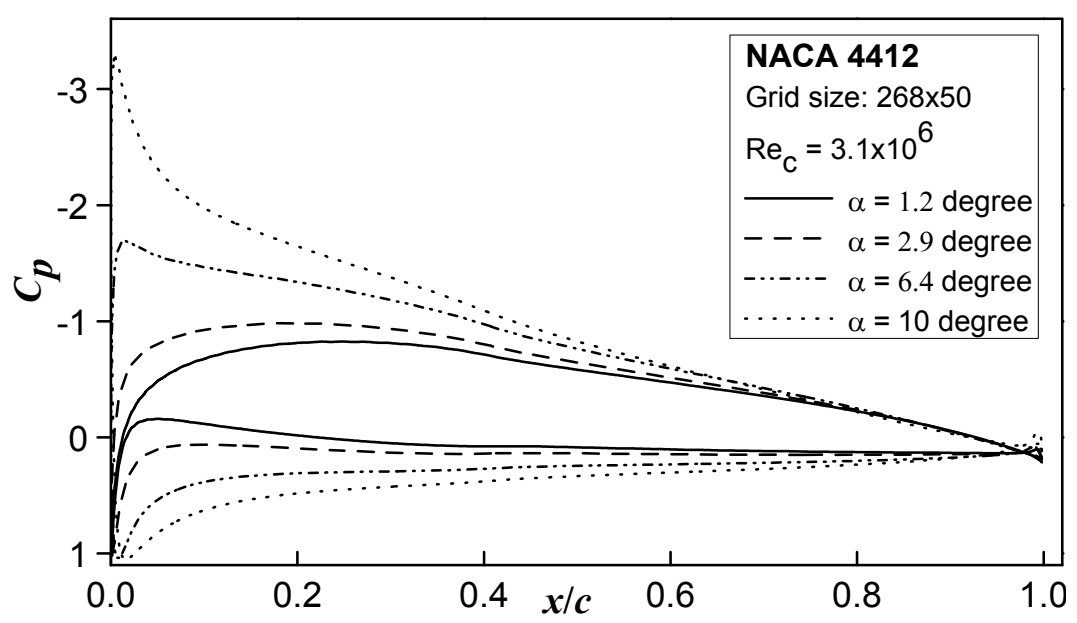

Fig.11. Effect of angle of attack on pressure coefficients for NACA 4412 hydrofoil. 


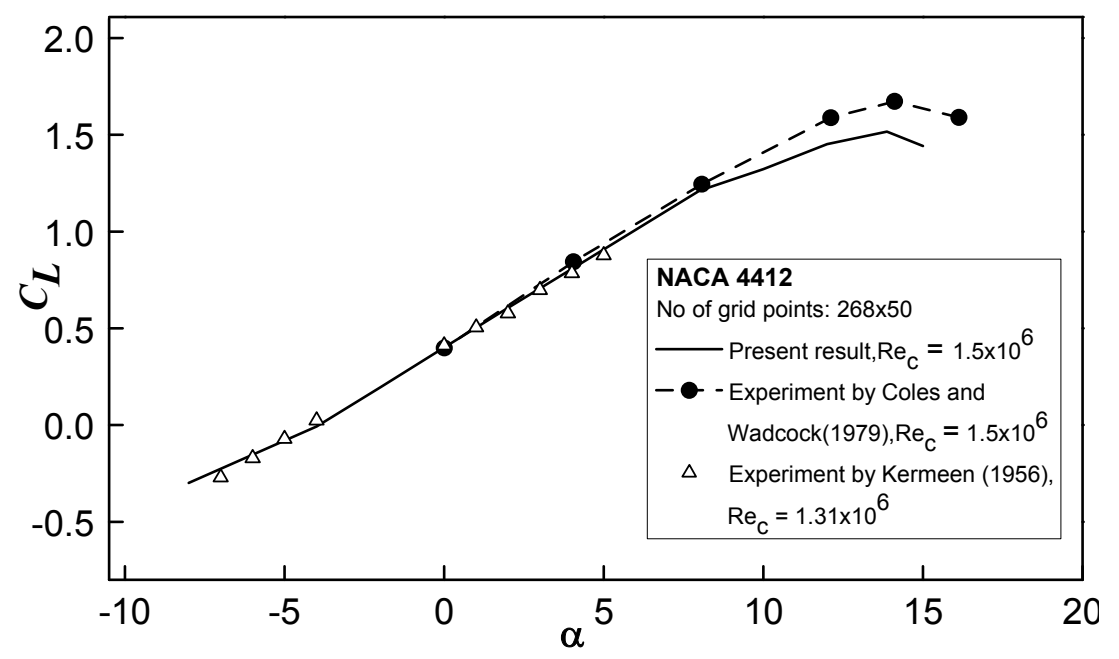

Fig.12. Lift coefficients for NACA 4412hydrofoil.

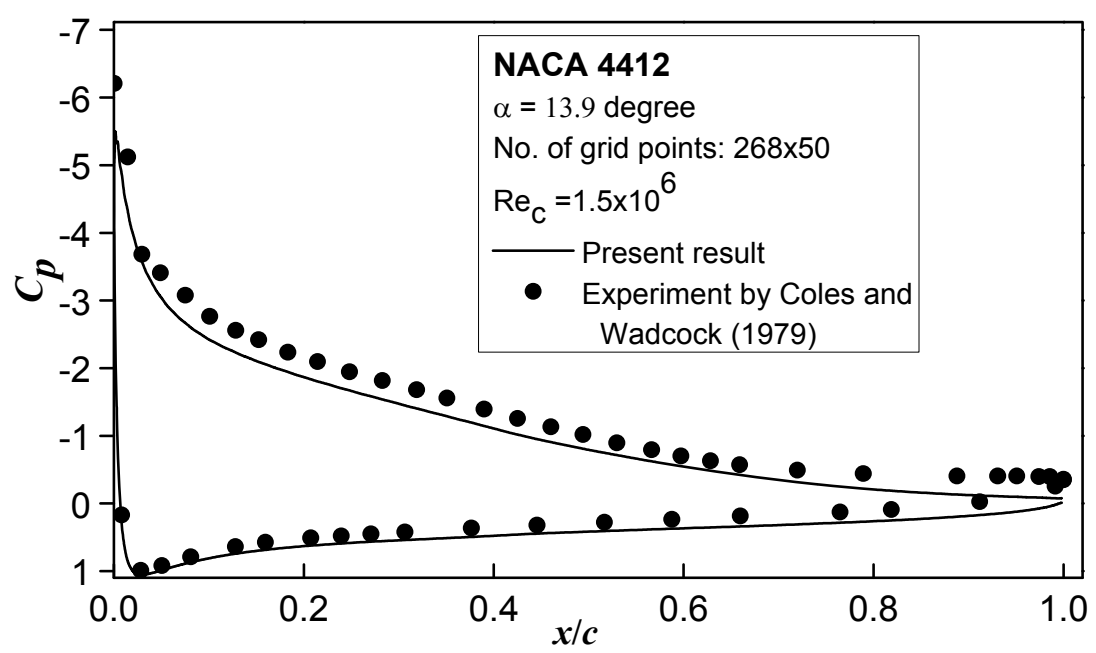

Fig.13. Pressure coefficients for NACA 4412 at 13.9 degree incidence.

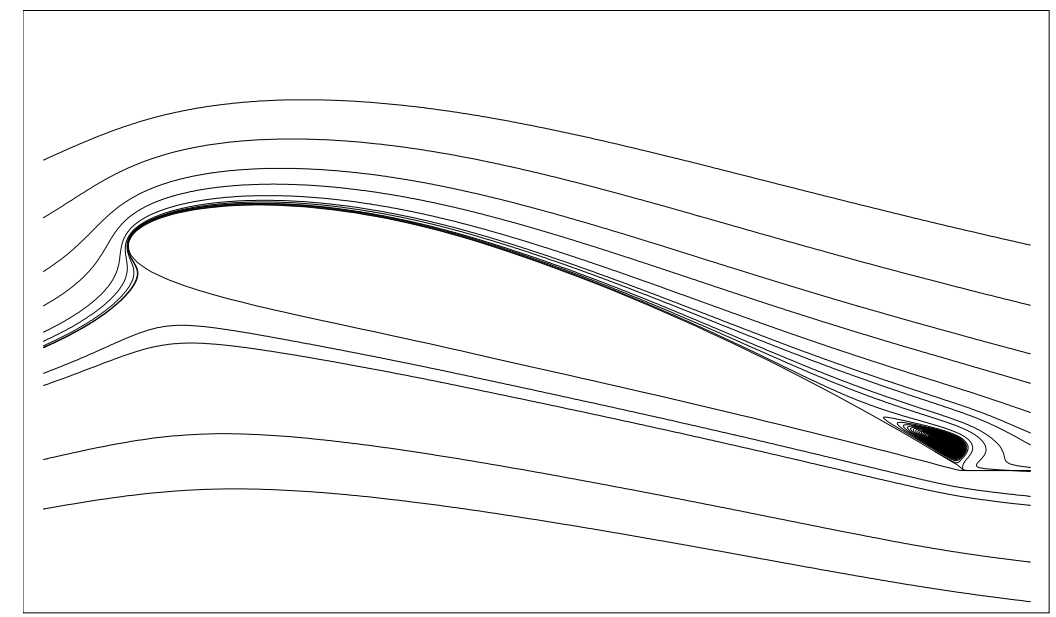

Fig.14. Streamlines for NACA 4412 at 13.9 degree incidence. 


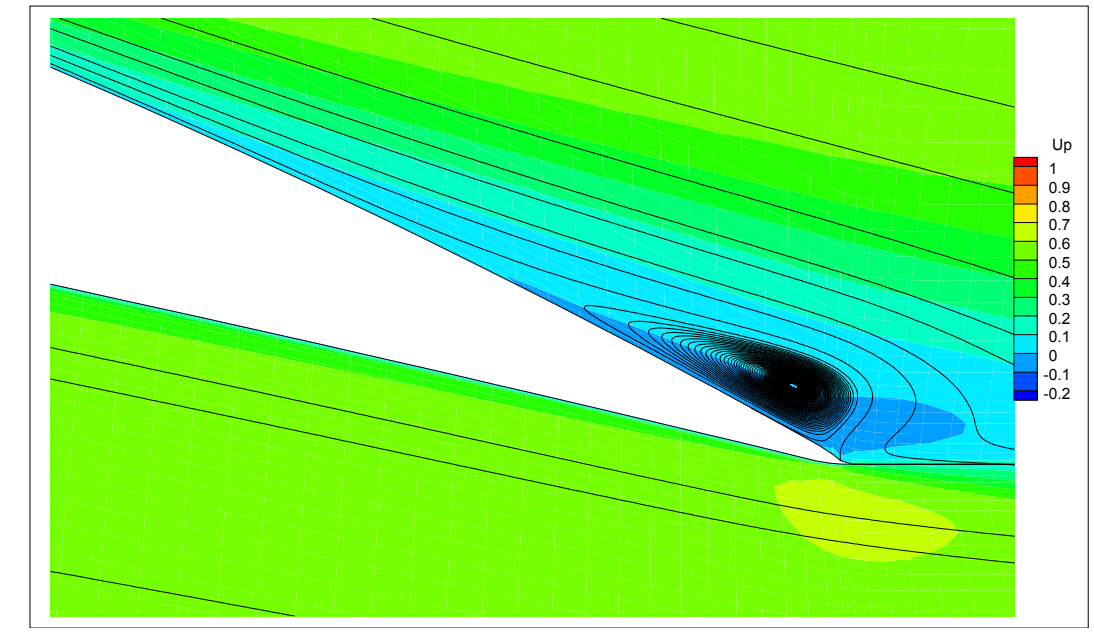

Fig.15. $u$-velocity contours for NACA 4412 at 13.9 deg incidence.

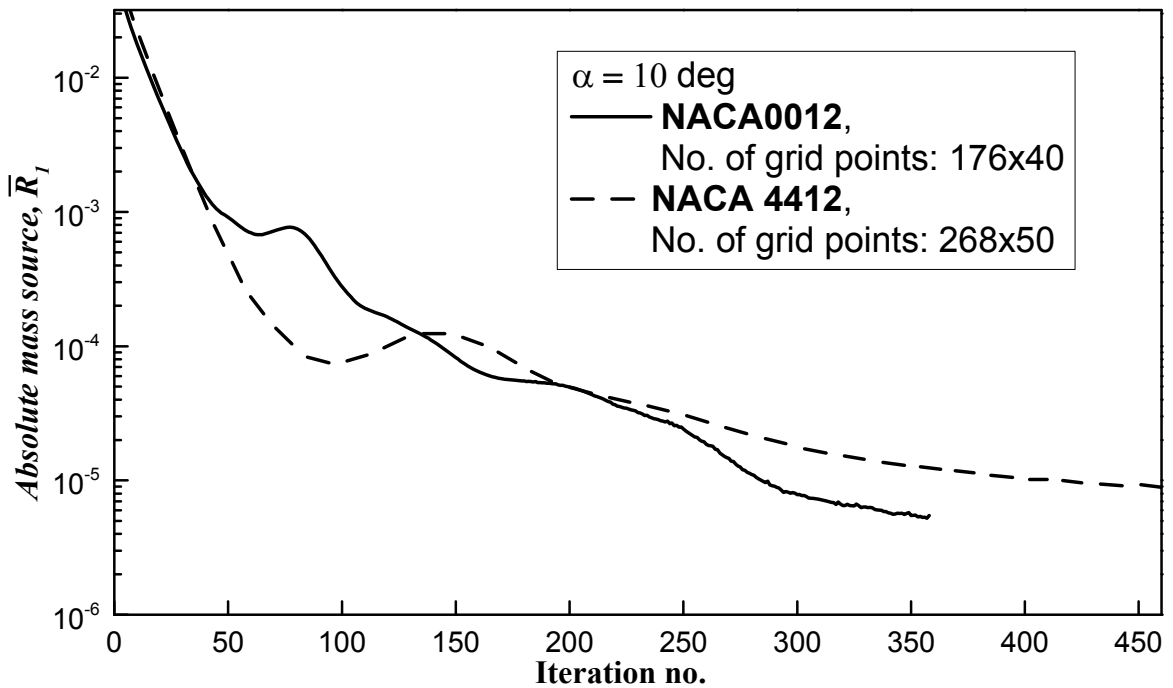

Fig.16. Convergence history.

\section{Conclusions}

This paper presents the numerical computation of turbulent flow past hydrofoils using the finite volume method with a $k-\varepsilon$ turbulence model. The following conclusions can be drawn from the present numerical study:

a) The surface pressure distributions on a number of hydrofoils such as NACA0012 [21] and NACA4412 located far from the free surface are computed and then compared with the theoretical as well as experimental data. The agreement with established data is found to be excellent except very close to the leading edge.

b) Grid refinement is carried out systematically keeping a proper distance between the first computational node and the wall. Significant improvement in results has been obtained with a refined grid particularly near the leading edge.

c) Despite simplifying the pressure correction equation by neglecting cross-diffusion contributions, proper under-relaxation factors may be used so that computational cost is reduced without adversely affecting the convergence rate. 
d) Lift coefficients are computed which exhibit an excellent agreement with experimental data at lower angles of attack. However, due to poor performance of the $k-\varepsilon$ turbulence model, at higher angles of attack when flow separation takes place, the agreement is found to be less satisfactory.

\section{Acknowledgement}

The authors are thankful to Dr. Milovan Peric for providing permission to use and modify the code CAFFA.

\section{Nomenclature}

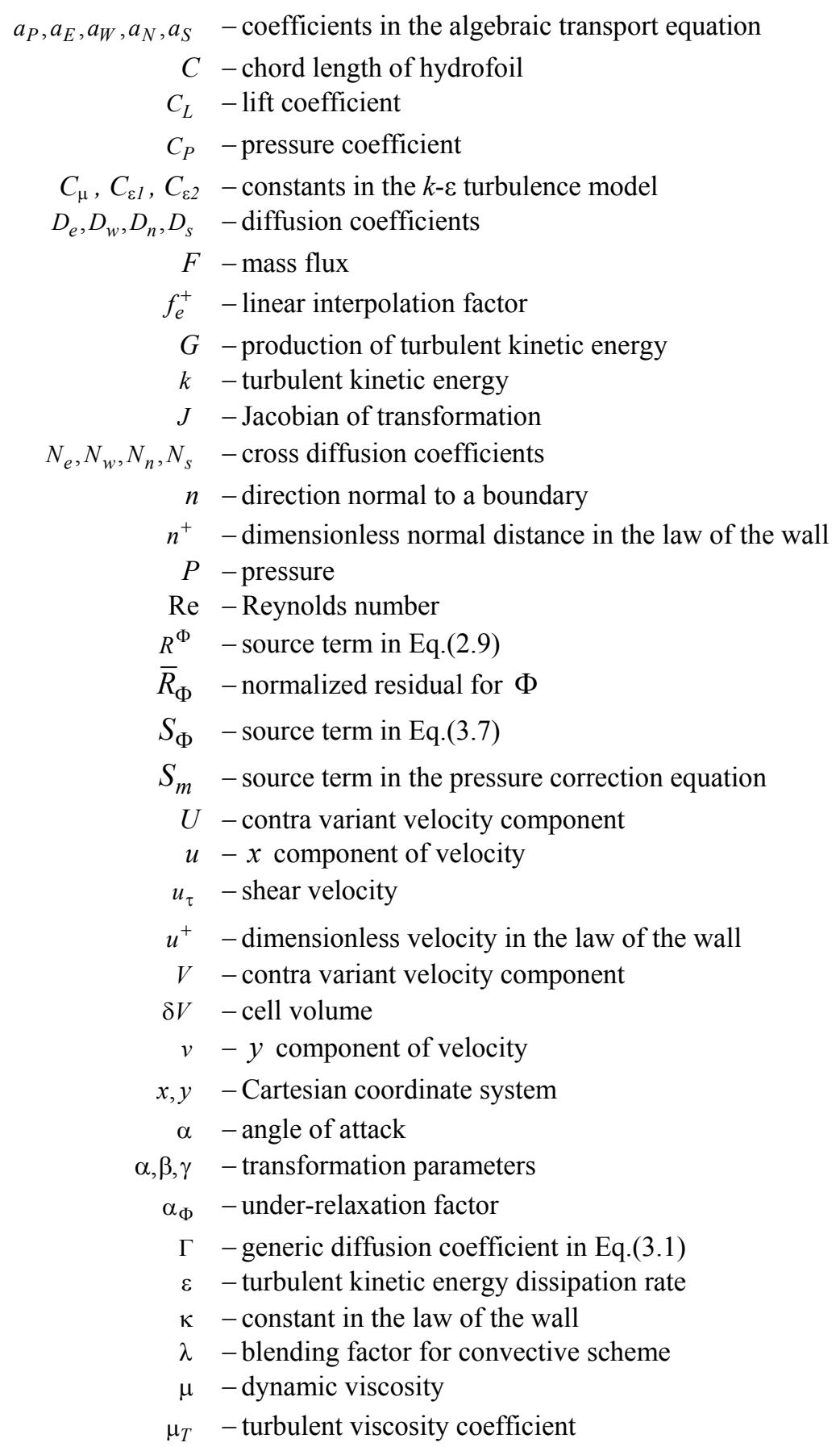




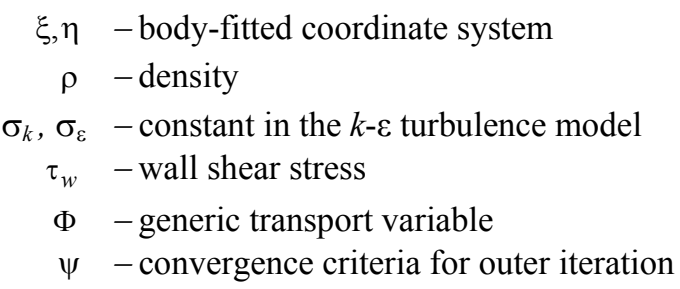

\section{References}

[1] Rhie C.M. (1981): A Numerical Study of the Flow Past an Isolated Airfoil with Separation. - PhD Thesis, Dept. of Mechanical and Industrial Engineering, University of Illinois at Urbana-Champaign.

[2] Peric M. (1985): A Finite Volume Method for the Prediction of Three-Dimensional Fluid Flow in Complex Ducts. - Ph.D. Thesis, University of London.

[3] Demirdzic I., Gosman A.D., Issa R.I. and Peric M. (1987): A calculation procedure for turbulent flow in complex geometries. - Computers and Fluids, vol.15, pp.251-273.

[4] Karki K.C and Patanker S.V. (1988): Calculation procedure for viscous incompressible flows in complex geometries. - Numerical Heat Transfer, vol.14, pp.295-307.

[5] Majumdar S. (1988): Role of under-relaxation in momentum interpolation for calculation of flow with nonstaggered grids. - Numerical Heat Transfer, Part B, vol.13, pp.125-132.

[6] Choi S.K. (1999): Note on the use of momentum interpolation method for unsteady flows. - Numerical. Heat Transfer, Part A, vol.36, pp.545-550.

[7] Masuko A. and Ogiwara S. (1990): Numerical simulation of viscous flow around practical Hull form. - Fifth International Conference on Numerical Ship Hydrodynamics, pp.211-224.

[8] Yu B., Tao W. and Wei J. (2002): Discussion on momentum interpolation method for collocated grids of incompressible flow. - Numerical Heat Transfer, Part B, vol.42, pp.141-166.

[9] Mulvany N., Tu J.Y., Chen L. and Anderson B. (2004): Assessment of two-equation turbulence modeling for high Reynolds number hydrofoil flow. - International Journal of Numerical Methods in Fluids, vol.45, pp.275-299.

[10] Kuzmin D. and Mierka O. (2006): On the implementation of the $k$ - $\varepsilon$ turbulence model in incompressible flow solvers based on a finite element discretization. - International Conference on Boundary and Interior Layers, Bail 2006, Germany, pp.1-8.

[11] Demirdzic I. (2015): On the discretization of diffusion term in finite-volume continuum mechanics. - Numerical Heat Transfer, Part B, vol.68, pp.1-10.

[12] Martınez J., Piscagliaa F., Montorfanoa A., Onoratia A. and Aithalb S.M. (2017): Influence of momentum interpolation methods on the accuracy and convergence of pressure-velocity coupling algorithms in Open FOAM. - Journal of Computational and Applied Mathematics, vol.309, pp.654-673.

[13] Patanker S.V. (1980): Numerical Heat Transfer and Fluid Flow. - New York: McGraw-Hill.

[14] Demirdzic I. and Peric M. (1990): Finite volume method for prediction of fluid flow in arbitrary shaped domains with moving boundary. - International Journal of Numerical Methods in Fluids, vol.10, pp.771-790.

[15] Peric M. (1990): Analysis of pressure-velocity coupling on non-orthogonal grids. - Numerical Heat Transfer, Part B, vol.17, pp.63-82.

[16] Patanker S.V. and Spalding D.B. (1972): A calculation procedure for heat, mass and momentum transfer in threedimensional parabolic flows. - International Journal of Heat and Mass Transfer, vol.15, pp.1787-1806.

[17] Stone H.L. (1968): Iterative solution of implicit approximations of multidimensional partial differential equations. - SIAM. Journal on Numerical Analysis, vol.5, pp.530-558. 
[18] Pinkerton R.M. (1936): Calculated and Measured Pressure Distributions over the Mid Span Section of the NACA 4412 Airfoil. - NACA Report No.563, National Advisory Committee for Aeronautics.

[19] Coles D. and Wadcock (1979): Flying-Hot-Wire Study of Flow Past an NACA 4412 Airfoil at Maximum Lift. AIAA Journal, vol.17, No.4, pp.321-329.

[20] Kermeen R.W. (1956): Water Tunnel Tests of NACA 4412 and WALCHNER PROFILE 7 Hydrofoils in Noncavitating and Cavitating flows. - California Institute of Technology, Hydrodynamics Laboratory, Report No. 47-5.

[21] Gregory N. and O'Reilly C.L. (1970): Low Speed Aerodynamic Characteristics of NACA 0012 Airfoil Section, Including the Effects of Upper Surface Roughness Simulation Hoarfrost. - National Physical Laboratory, Teddington, England, Aero Report No.1308.

Received: November 21, 2017

Revised: June 23, 2018 